\title{
On solving periodic Riccati equations
}

\author{
A. $\operatorname{Varga} a^{*} \dagger$ \\ German Aerospace Center, DLR-Oberpfaffenhofen, Institute of Robotics and Mechatronics, \\ D-82234 Wessling, Germany
}

\begin{abstract}
SUMMARY
Numerically reliable algorithms to compute the periodic non-negative definite stabilizing solutions of the periodic differential Riccati equation (PRDE) and discrete-time periodic Riccati equation (DPRE) are proposed. For the numerical solution of PRDEs, a new multiple shooting-type algorithm is developed to compute the periodic solutions in an arbitrary number of time moments within one period by employing suitable discretizations of the continuous-time problems. In contrast to single shooting periodic generator methods, the multiple shooting-type methods have the main advantage of being able to address problems with larger periods. Three methods are discussed to solve DPREs. Two of the methods represent extensions of the periodic QZ algorithm to non-square periodic pairs, whereas the third method represents an extension of a quotient-product swapping and collapsing 'fast' algorithm. All proposed approaches are completely general, being applicable to periodic Riccati equations with time-varying dimensions as well as with singular weighting matrices. Copyright (C) 2008 John Wiley \& Sons, Ltd.
\end{abstract}

Received 17 March 2008; Accepted 18 March 2008

KEY WORDS: periodic systems; periodic Riccati equation; periodic Lyapunov equation; periodic deadbeat control; numerical methods

\section{INTRODUCTION}

In this paper we consider the numerical solution of the periodic Riccati differential equation (PRDE):

$$
-\dot{X}(t)=A^{\mathrm{T}}(t) X(t)+X(t) A(t)+C^{\mathrm{T}}(t) C(t)-X(t) B(t) B^{\mathrm{T}}(t) X(t)
$$

\footnotetext{
*Correspondence to: A. Varga, German Aerospace Center, DLR-Oberpfaffenhofen, Institute of Robotics and Mechatronics, D-82234 Wessling, Germany.

${ }^{\dagger}$ E-mail: Andras.Varga@dlr.de

Contract/grant sponsor: Swedish Strategic Research Foundation
}

Copyright (C) 2008 John Wiley \& Sons, Ltd. 


\section{A. VARGA}

where $A(t), B(t), C(t)$ are $n \times n, n \times m, p \times n$, and $m \times m$ real $T$-periodic matrices (i.e. $A(t+T)=$ $A(t), B(t+T)=B(t), C(t+T)=C(t), \forall t)$ and integrable over [0,T]. The PRDE plays a major role in solving the optimal periodic linear-quadratic (PLQ) control problem [1] for the linear continuous-time periodic system

$$
\begin{aligned}
& \dot{x}(t)=A(t) x(t)+B(t) u(t) \\
& y(t)=C(t) x(t)
\end{aligned}
$$

with the associated quadratic cost functional

$$
J=\frac{1}{2} \int_{0}^{\infty}\left[x^{\mathrm{T}}(t) u^{\mathrm{T}}(t)\right]\left[\begin{array}{cc}
Q(t) & S(t) \\
S^{\mathrm{T}}(t) & R(t)
\end{array}\right]\left[\begin{array}{l}
x(t) \\
u(t)
\end{array}\right] \mathrm{d} t
$$

where $Q(t)=C^{\mathrm{T}}(t) C(t), S(t)$ is an $n \times m \quad T$-periodic matrix, and $R(t)=R^{\mathrm{T}}(t)>0$ is an $m \times m T$-periodic matrix. Usually it is assumed that $Q(t)-S^{\mathrm{T}}(t) R^{-1}(t) S(t) \geqslant 0$. Minimizing $J$ to find the optimal control $u^{*}(t)$ can be recast into solving, for the stabilizing positivesemidefinite $T$-periodic solution $X(t)$, a PRDE of form (1) with $A(t), B(t)$, and $C(t)$ replaced by $\widetilde{A}(t)=A(t)-B(t) R^{-1}(t) S(t), \widetilde{B}(t)$ and $\widetilde{C}(t)$, respectively, where $\widetilde{B}(t)$ and $\widetilde{C}(t)$ are defined by the full-rank Cholesky-like factorizations $\widetilde{B}(t) \widetilde{B}^{\mathrm{T}}(t)=B(t) R^{-1}(t) B^{\mathrm{T}}(t)$ and $\widetilde{C}^{\mathrm{T}}(t) \widetilde{C}(t)=$ $Q(t)-S^{\mathrm{T}}(t) R^{-1}(t) S(t)$. The optimal periodic state-feedback control law $u^{*}(t)=F(t) x(t)$ with

$$
F(t)=-R^{-1}(t)(X(t) B(t)+S(t))^{\mathrm{T}}
$$

minimizes the performance index (3) and ensures that $A(t)+B(t) F(t)$ is asymptotically stable.

We will also be concerned with the numerical solution of the discrete-time periodic Riccati equation (DPRE):

$$
X_{k}=C_{k}^{\mathrm{T}} C_{k}+A_{k}^{\mathrm{T}} X_{k+1} A_{k}-\left(A_{k}^{\mathrm{T}} X_{k+1} B_{k}+S_{k}\right)\left(R_{k}+B_{k}^{\mathrm{T}} X_{k+1} B_{k}\right)^{-1}\left(A_{k}^{\mathrm{T}} X_{k+1} B_{k}+S_{k}\right)^{\mathrm{T}}
$$

where $A_{k} \in \mathbb{R}^{n_{k+1} \times n_{k}}, \quad B_{k} \in \mathbb{R}^{n_{k+1} \times m_{k}}, \quad C_{k} \in \mathbb{R}^{p_{k} \times n_{k}}, \quad R_{k}=R_{k}^{\mathrm{T}} \in \mathbb{R}^{m_{k} \times m_{k}}$, and $S_{k} \in \mathbb{R}^{n_{k} \times m_{k}}$ are $N$-periodic matrices (i.e. $A_{k+N}=A_{k}, B_{k+N}=B_{k}, C_{k+N}=C_{k}, R_{k+N}=R_{k}, S_{k+N}=S_{k}, \forall k$ ). Equation (5) arises, for example, when solving the PLQ optimal control problem [1] for the linear discrete-time periodic system

$$
\begin{aligned}
x_{k+1} & =A_{k} x_{k}+B_{k} u_{k} \\
y_{k} & =C_{k} x_{k}
\end{aligned}
$$

by minimizing the quadratic cost functional

$$
J=\frac{1}{2} \sum_{k=0}^{\infty}\left[\begin{array}{ll}
x_{k}^{\mathrm{T}} & u_{k}^{\mathrm{T}}
\end{array}\right]\left[\begin{array}{ll}
Q_{k} & S_{k} \\
S_{k}^{\mathrm{T}} & R_{k}
\end{array}\right]\left[\begin{array}{l}
x_{k} \\
u_{k}
\end{array}\right]
$$

where $Q_{k}=C_{k}^{\mathrm{T}} C_{k}$ and usually it is assumed that

$$
\left[\begin{array}{ll}
Q_{k} & S_{k} \\
S_{k}^{\mathrm{T}} & R_{k}
\end{array}\right] \geqslant 0
$$


Provided a non-negative $N$-periodic stabilizing solution $X_{k}$ of the DPRE (5) is known, the periodic state-feedback matrix $F_{k}$ in the optimal control law $u_{k}^{*}=F_{k} x_{k}$, which minimizes the performance index (7), results as

$$
F_{k}=-\left(B_{k}^{\mathrm{T}} X_{k+1} B_{k}+R_{k}\right)^{-1}\left(A_{k}^{\mathrm{T}} X_{k+1} B_{k}+S_{k}\right)^{\mathrm{T}}
$$

The dual equations

$$
\dot{X}(t)=A(t) X(t)+X(t) A^{\mathrm{T}}(t)+B(t) B^{\mathrm{T}}(t)-X(t) C^{\mathrm{T}}(t) C(t) X(t)
$$

and

$$
X_{k+1}=B_{k} B_{k}^{\mathrm{T}}+A_{k} X_{k} A_{k}^{\mathrm{T}}-\left(A_{k} X_{k} C_{k}^{\mathrm{T}}+S_{k}\right)\left(R_{k}+C_{k} X_{k} C_{k}^{\mathrm{T}}\right)^{-1}\left(A_{k} X_{k} C_{k}^{\mathrm{T}}+S_{k}\right)^{\mathrm{T}}
$$

arise in the optimal filtering problems [1,2]. A particular case of PRDE corresponding to the case when $B(t) \equiv 0$ is the periodic Lyapunov differential equation (PLDE):

$$
-\dot{X}(t)=A^{\mathrm{T}}(t) X(t)+X(t) A(t)+Q(t)
$$

where $Q(\cdot)$ is symmetrical and $T$-periodic. This equation together with its dual

$$
\dot{X}(t)=A(t) X(t)+X(t) A^{\mathrm{T}}(t)+Q(t)
$$

have many important applications in the analysis of reachability/observability of linear continuoustime periodic systems [3], in solving periodic stabilization problems [4], in computing Hankeland $\mathscr{H}_{2}$-norms of periodic systems [2,5], or in solving PRDEs by employing Newtons' method [1].

This paper deals with the efficient and numerically reliable computation of the unique symmetric stabilizing periodic solutions of the PRDE and DPRE. For the numerical solution of the dual equations (9) and (10), the same methods can be applied to appropriate dual reformulations. The paper relies on the preliminary results reported in [5] for solving PRDEs and in [6] for solving DPREs.

To compute the $T$-periodic solution $X(t)$ of Equation (1), traditionally the periodic generator method is used (see, for example, [7]). Essentially, this single shooting-type method computes an initial condition matrix $X_{0}$ (also called a periodic generator) such that the integration of the ordinary differential equation (ODE) (1) with the initial value $X(0)=X_{0}$ leads to a $T$-periodic solution $X(t)$ satisfying $X(T)=X_{0}$. Unfortunately, this approach is known to be highly sensitive due to the ill-conditioning of the underlying Hamiltonian-ODE problem to be solved (due to the intrinsic unstable dynamics), especially in the case of large periods. Therefore, in this paper we propose a numerically enhanced multiple shooting-type approach based on employing discretization techniques. The main appeal of this approach is that the periodic solution $X(t)$ is computed simultaneously in many time moments within one period, so that the numerical difficulties related to numerical integrations for large periods and/or unstable dynamics are highly alleviated. The solution for intermediate values of time $t$ can be computed using special numerical integration by initializing the solution in the nearest time moment. The key numerical element for solving 


\section{A. VARGA}

the discrete-time problem is the ordered periodic real Schur form (PRSF) of a symplectic matrix product together with the computation of the corresponding periodic invariant bases of the stable periodic invariant subspace $[8,9]$.

The solution of DPRE for constant dimensions has been considered by several authors [8-10], but DPREs with time-varying dimensions have been considered only recently in [11]. A standard assumption in all proposed algorithms is the invertibility of $R_{k}$. The methods proposed in $[8,9]$ rely on the periodic QZ decomposition to compute orthogonal bases for suitable periodic deflating subspaces from which the solution results. The main numerical element for these methods is the computation of an ordered periodic QZ decomposition of a pair of periodic square matrices with constant dimensions. Algorithms to perform this decomposition have been proposed in $[8,9]$, but presently there is no robust numerical software implementing these methods for pairs of real periodic matrices [12].

The methods proposed in $[10,11]$ belong to the family of 'fast' methods, by reducing the problem to a single discrete-time algebraic Riccati equation (DARE) satisfied say by $X_{1}=X_{N+1}$. This DARE can then be solved using standard deflating subspace methods. The rest of the solution $X_{k}$ for $k=N, N-1, \ldots, 2$, can be obtained by a convergent direct iteration on (5) [13]. The quotient-product swapping and collapsing method of $[10,14]$ has been reinterpreted to perform locally orthogonal compressions on an extended regular pencil to obtain a subpencil whose stable deflating subspace generates the stabilizing solution at a certain time moment [15]. This method performs substantially less operations than the periodic QZ-decomposition-based approach, and this justifies the term 'fast' used to label it [15]. The method of [11] performs essentially the same reduction of the extended pencil by using structure preserving non-orthogonal block compressions. In the resulting final subpencil, the 'matrices' of a standard problem can be identified, which define the solution at a certain time moment. To compute the solution, a structure-preserving iterative doubling algorithm with quadratic convergence is employed in [11], but any alternative approach for standard systems can be employed as well.

In this paper, three extensions of the above-described methods are proposed, which can address the most general case of time-varying dimensions and singular weighting matrices $R_{k}$. DPREs with possibly time-varying dimensions are to be expected when solving spectral and inner-outer factorization problems for periodic systems using deflation-based approaches similar to those proposed in [16] for standard systems. Problems with singular $R_{k}$ arise when solving, for example, deadbeat control problems via an PLQ-optimization-based approach [17]. All three proposed methods are able to address problems with varying number of null characteristic multipliers of $A_{k}$. The first two methods extend the periodic QZ algorithm to handle non-square periodic pairs with time-varying dimensions. To apply the standard periodic QZ algorithm, a certain periodic pair is preprocessed by first deflating its infinite characteristic multipliers and then isolating the core finite characteristic multipliers. The first method performs an explicit finite-infinite separation of characteristic spectra, whereas the second method employs dedicated deflation techniques to separate the trivial zero and infinite characteristic multipliers originating from the time-varying dimensions. The third method performs structure exploiting orthogonal transformations to reduce an extended regular pencil to a regular subpencil, whose deflating subspaces produce the solution of DPRE at a fixed time moment. This method extends the quotient-product swapping and collapsing approach of [10] along the lines of the reduction technique employed in [18]. The main advantage of this approach is that its implementation is straightforward using available robust numerical software. For the 'fast' method, reliable software implementation is already available in a recently developed Periodic Systems Toolbox [19]. 


\section{PERIODIC RICCATI EQUATIONS}

\section{SOLUTION OF PRDE}

In this section we address the computation of the periodic stabilizing solution of the PRDE (1) and of the periodic solution of the PLDE (11).

\subsection{Existence results}

First, we introduce some basic concepts for continuous-time periodic systems. Let $\Phi_{A}(t, \tau)$ denote the transition matrix corresponding to $A(t)$ satisfying

$$
\frac{\partial \Phi_{A}(t, \tau)}{\partial t}=A(t) \Phi_{A}(t, \tau), \quad \Phi_{A}(\tau, \tau)=I
$$

For a $T$-periodic $A(t), \Psi_{A}(\tau):=\Phi_{A}(\tau+T, \tau)$ is called the monodromy matrix corresponding to $A(t)$, and its eigenvalues, which are independent of $\tau$, are called characteristic multipliers. We say that $A(t)$ is a stable periodic matrix if all its characteristic multipliers have magnitudes less than 1 .

To provide the main existence result, we recall the notions of reachability/stabilizability and observability/detectability of periodic systems (see, for example, [1]). A characteristic multiplier $\lambda$ of $A(t)$ is said to be unreachable if $\Psi_{A}^{\mathrm{T}}(0) x=\lambda x, x \neq 0$, imply that $B^{\mathrm{T}}(t) \Phi_{A}^{\mathrm{T}}(0, t) x=0$, almost everywhere for $t \in[0, T]$. A characteristic multiplier $\lambda$ of $A(t)$ is said to be reachable if it is not unreachable. System (2) is said to be stabilizable if all characteristic multipliers $\lambda$ with $|\lambda| \geqslant 1$ are reachable. Similarly, a characteristic multiplier $\lambda$ of $A(t)$ is said to be unobservable if $\Psi_{A}(0) x=\lambda x$ and $x \neq 0$ imply that $C(t) \Phi_{A}(t, 0) x=0$, almost everywhere for $t \in[0, T]$. A characteristic multiplier $\lambda$ of $A(t)$ is said to be observable if it is not unobservable. System (2) is said to be detectable if all characteristic multipliers $\lambda$ with $|\lambda| \geqslant 1$ are observable.

A solution $X(t)$ of (1) is called a stabilizing solution if $A(t)-B(t) B^{\mathrm{T}}(t) X(t)$ is stable. The following result from [20] gives necessary and sufficient conditions for the existence of a unique symmetric positive-semidefinite stabilizing solution:

\section{Theorem 1}

The PRDE (1) admits a unique $T$-periodic stabilizing solution $X(t)=X^{\mathrm{T}}(t) \geqslant 0$ if and only if the pair $(A(t), B(t))$ is stabilizable and the pair $(A(t), C(t))$ is detectable.

A less restrictive assumption guarantees the existence of a (possibly non-unique) stabilizing symmetric positive-semidefinite stabilizing solution [1]:

\section{Theorem 2}

The PRDE (1) admits a $T$-periodic stabilizing solution $X(t)=X^{\mathrm{T}}(t) \geqslant 0$ if and only if the pair $(A(t), B(t))$ is stabilizable and no unit-modulus characteristic multiplier of $A(t)$ is unobservable.

This theorem is applicable in the important case $C(t) \equiv 0$.

If $B(t) \equiv 0$, the PRDE becomes a PLDE for which the existence result has a particularly simple form:

\section{Theorem 3}

The PLDE (11) admits a unique $T$-periodic solution $X(t)$ if and only if $A(t)$ does not have reciprocal characteristic multipliers. 


\section{A. VARGA}

In what follows, we will tacitly assume that the appropriate existence conditions are always fulfilled when solving particular PRDEs.

\subsection{Periodic generator method}

Let $H(t)$ be the Hamiltonian matrix corresponding to the PRDE (1):

$$
H(t)=\left[\begin{array}{cc}
A(t) & -B(t) B^{\mathrm{T}}(t) \\
-C^{\mathrm{T}}(t) C(t) & -A^{\mathrm{T}}(t)
\end{array}\right]
$$

We have $J H(t)+H^{\mathrm{T}}(t) J=0$, where

$$
J=\left[\begin{array}{cc}
O & I \\
-I & O
\end{array}\right]
$$

thus, $H(t)$ is indeed a Hamiltonian matrix. Theorems 1 and 2 guarantee that the monodromy matrix $\Psi_{H}(0)$ is symplectic (i.e. $\Psi_{H}^{\mathrm{T}}(0) J \Psi_{H}(0)=J$ ), with $2 n$ eigenvalues appearing in pairs of the form $\left(\lambda_{i}, 1 / \lambda_{i}\right)$, for $i=1, \ldots, n$. This property is the key aspect of the following approach to solve the PRDE (1) (see [7]):

1. Compute the symplectic monodromy matrix $\Psi_{H}(0)$.

2. Compute orthogonal $Z$ to reduce $\Psi_{H}(0)$ to an ordered real Schur form (RSF) such that

$$
Z^{\mathrm{T}} \Psi_{H}(0) Z=\left[\begin{array}{cc}
\Theta_{11} & \Theta_{12} \\
O & \Theta_{22}
\end{array}\right]
$$

where $\Theta_{11}$ has $n$ eigenvalues inside the unit circle and $\Theta_{22}$ has $n$ eigenvalues outside the unit circle.

3. Partition $Z$ into $n \times n$ blocks

$$
Z=\left[\begin{array}{ll}
Z_{11} & Z_{12} \\
Z_{21} & Z_{22}
\end{array}\right]
$$

and integrate from $t=0$ to $t=T$ the linear matrix differential equation:

$$
\dot{S}(t)=H(t) S(t), \quad S(0)=\left[\begin{array}{l}
Z_{11} \\
Z_{21}
\end{array}\right]
$$

From the conformably partitioned solution

$$
S(t)=\left[\begin{array}{l}
S_{1}(t) \\
S_{2}(t)
\end{array}\right]
$$

compute $X(t)=S_{2}(t) S_{1}^{-1}(t)$.

Alternatively, it is possible to determine the periodic generator $X_{0}:=X(0)$ by solving the algebraic Riccati equation [3]:

$$
\Psi_{21}+\Psi_{22} X_{0}-X_{0} \Psi_{11}-X_{0} \Psi_{12} X_{0}=0
$$


where

$$
\Psi_{H}(0)=\left[\begin{array}{ll}
\Psi_{11} & \Psi_{12} \\
\Psi_{21} & \Psi_{22}
\end{array}\right]
$$

Then, the solution on $[0, T]$ can be computed by numerical integration of the PRDE (9).

Note that the same approaches can be employed to solve the dual PRDE (9) by defining

$$
H(t)=\left[\begin{array}{cc}
-A^{\mathrm{T}}(t) & B(t) B^{\mathrm{T}}(t) \\
C^{\mathrm{T}}(t) C(t) & A(t)
\end{array}\right]
$$

To solve the PLDE (11), we determine the periodic generator $X_{0}:=X(0)$ by solving the standard discrete-time Lyapunov equation satisfied by $X(T)(=X(0))$ [3]

$$
X_{0}=\Psi_{A}^{\mathrm{T}}(0) X_{0} \Psi_{A}(0)+W(T, 0)
$$

where

$$
W\left(t_{f}, t\right):=\int_{t}^{t_{f}} \Phi_{A}^{\mathrm{T}}(\tau, t) Q(\tau) \Phi_{A}(\tau, t) \mathrm{d} \tau
$$

and then integrate (11) backward in time from $t=T$ to $t=0$.

The periodic generator approach to solve PRDEs is potentially numerically unreliable because it involves the numerical integration of one or two ODEs with unstable dynamics: the first to compute $\Psi_{H}(0)$ and the second to compute $S(t)$. Therefore, for large periods, this approach will almost certainly fail because of the uncontrolled accumulation of roundoff errors. The second approach will encounter similar difficulties for large periods because of the need to compute $\Psi_{H}(0)$.

\subsection{Multiple shooting method to solve PRDE}

To alleviate the numerical difficulties related to the periodic generator method, we propose an alternative approach that relies on determining the transition matrix $\Phi_{H}(T, 0)$ in a product form (also recommended in [21])

$$
\Phi_{H}(T, 0)=\Phi_{H}(T, T-\Delta) \ldots \Phi_{H}(2 \Delta, \Delta) \Phi_{H}(\Delta, 0)
$$

where $\Delta=T / N$ for a suitably chosen integer period $N$. We denote $H_{k}:=\Phi_{H}(k \Delta,(k-1) \Delta)$ for $k=1,2, \ldots$, which is obviously an $N$-periodic matrix. Using the algorithm of [8], we can determine an orthogonal $N$-periodic matrix $Z_{k}$ to reduce $H_{k}$ to an ordered PRSF such that

$$
Z_{k+1} H_{k} Z_{k}=\left[\begin{array}{cc}
J_{k ; 11} & J_{k ; 12} \\
O & J_{k ; 22}
\end{array}\right]
$$

where $\Theta_{11}:=J_{N ; 11 \ldots J_{2 ; 11}} J_{1 ; 11}$ has $n$ eigenvalues inside the unit circle and $\Theta_{22}:=J_{N ; 22} \ldots$ $J_{2 ; 22} J_{1 ; 22}$ has $n$ eigenvalues outside the unit circle. Since $\Psi_{H}(0)=H_{N} \ldots H_{2} H_{1}$, it follows that $Z_{1}^{\mathrm{T}} \Psi_{H}(0) Z_{1}$ is in the ordered RSF (14), where both $\Theta_{11}$ and $\Theta_{22}$ are in RSF, and $Z$ is simply $Z_{1}$. If we partition $Z_{k}$ into $n \times n$ blocks as

$$
Z_{k}=\left[\begin{array}{cc}
Z_{k ; 11} & Z_{k ; 12} \\
Z_{k ; 21} & Z_{k ; 22}
\end{array}\right]
$$




\section{A. VARGA}

we obtain the solution of PRDE at $t=(k-1) \Delta$ as

$$
X_{k}:=X((k-1) \Delta)=Z_{k ; 21} Z_{k ; 11}^{-1}
$$

Some computational aspects are relevant for a robust implementation of this approach. To compute $H_{k}=\Phi_{H}(k \Delta,(k-1) \Delta)$, we have to integrate the ODE

$$
\dot{Y}(t)=H(t) Y(t), \quad Y((k-1) \Delta)=I
$$

for $t \in[k \Delta,(k-1) \Delta]$ to obtain $H_{k}=Y(k \Delta)$. Since each matrix $H_{k}$ is symplectic, it is important to employ numerical integrators that are able to guarantee this property. Note that standard methods (even the simple explicit Euler method) do not ensure that $H_{k}$ will be symplectic. Among methods able to compute symplectic solutions are the Gauss-Legendre (diagonal Padé approximants) methods, which belong to the class of symplectic Runge-Kutta methods [7, 22]. MATLAB software for symplectic integration is freely available [23].

We can also apply the fast algorithm for DPRE (see the following section) by observing that the solution $X(t)$ at two successive time steps $(k-1) \Delta$ and $k \Delta$ is related as [3]

$$
X_{k}=\left(X_{k+1} H_{k, 12}-H_{k, 22}\right)^{-1}\left(H_{k, 21}-X_{k+1} H_{k, 11}\right)
$$

where $H_{k}$ is partitioned into $n \times n$ blocks

$$
H_{k}=\left[\begin{array}{ll}
H_{k, 11} & H_{k, 12} \\
H_{k, 21} & H_{k, 22}
\end{array}\right]
$$

Rewriting the above expression of $X_{k}$, we obtain

$$
H_{k}\left[\begin{array}{c}
I \\
X_{k}
\end{array}\right]=\left[\begin{array}{c}
I \\
X_{k+1}
\end{array}\right]\left(H_{k, 11}+H_{k, 12} X_{k}\right)
$$

which, with $M_{k}:=H_{k}$ and $L_{k}:=I$, is in form (24), which underlies the methods discussed in the following section for solving DPRE. If $Z_{1}$, computed by the fast algorithm, is partitioned into form (19), then $X_{1}=Z_{1,21} Z_{1,11}^{-1}$. The rest of the solution $X_{k}$ for $k=N, \ldots, 2$ is computed iteratively using (20), with $X_{N+1}:=X_{1}$. The main advantage of this method is its ease of implementation, since no sophisticated methods (such as algorithms to compute ordered PRSF) are involved.

To compute the values of the solution $X(t)$ between two discretization moments $t_{0}=(k-1) \Delta$ and $t_{f}=k \Delta$, special ODE solvers as those proposed in [24,25] can be used to integrate (1) in backward time with $X\left(t_{f}\right)=X(k \Delta)$ or (9) in forward time with $X\left(t_{0}\right)=X((k-1) \Delta)$. A distinctive feature of solvers discussed in [25] is their capability to preserve the positivity of the numerical solution of differential Riccati equations.

The computational approach involves as preprocessing and postprocessing steps the numerical integration of the underlying PRDE on small time intervals. These parts of computations are usually the most computer intensive and we assumed tacitly that existing standard or symplectic integration techniques can be used to perform them. Fortunately, it appears that these computations 
PERIODIC RICCATI EQUATIONS

are 'embarrassingly' parallelizable, which makes the multiple shooting approach very appealing on parallel machines.

\subsection{Multiple shooting method to solve PLDE}

To solve the PLDE (11) using a multiple shooting-type approach, we observe that the solution $X(t)$ at time moments $t$ and $t-\Delta$ is related as [3]

$$
X(t-\Delta)=\Phi_{A}^{\mathrm{T}}(t, t-\Delta) X(t) \Phi_{A}(t, t-\Delta)+W(t, t-\Delta)
$$

where $W(t, t-\Delta)$ is defined in (16). Thus, the solution at successive time moments $(k-1) \Delta$ and $k \Delta$ satisfies

$$
X_{k}=F_{k}^{\mathrm{T}} X_{k+1} F_{k}+W_{k}
$$

where $F_{k}:=\Phi_{A}(k \Delta,(k-1) \Delta)$ and $W_{k}:=W(k \Delta,(k-1) \Delta)$. By imposing $X_{N+1}=X_{1}$, the $N$-coupled equations in (21) for $k=1, \ldots, N$ represent a discrete-time backward periodic Lyapunov equation.

To solve the periodic discrete-time Lyapunov equation (21), a numerically reliable method, such as that proposed in [26], can be used. This method is based on computing the PRSF of the periodic matrix $F_{k}$. For the computation of PRSF, numerically stable algorithms have been proposed in $[8,9]$ and robust numerical software implementations are available in the recently developed Periodic Systems Toolbox for MATLAB [19]. For the solution of the periodic Lyapunov equation (21), robust numerical software is also available in this toolbox. A similar approach can be used to solve the dual PLDE (12).

The computation of $F_{k}$ and $W_{k}$ for $k=1, \ldots, N$ in (21) can be done using numerical integration of appropriate ODEs. To compute $F_{k}$, the matrix differential equation (13) must be integrated from $\tau=$ $(k-1) \Delta$ to $k \Delta$ using appropriate methods for ODEs. Since the time step $\Delta$ can be chosen arbitrarily small, the numerical integration even for unstable $A(t)$ will not raise any numerical difficulties.

To compute $W_{k}$ observe that for given $t_{f}, Y(t):=W\left(t_{f}, t\right)$ in (16) satisfies the Lyapunov differential equation:

$$
-\dot{Y}(t)=A(t) Y(t)+Y(t) A^{\mathrm{T}}(t)+Q(t), \quad Y\left(t_{f}\right)=0
$$

Thus, $W_{k}$ can be computed as $W_{k}=Y((k-1) \Delta)$ by integrating the above equation backward in time from $t_{f}=k \Delta$ to $t_{0}=(k-1) \Delta$. To integrate the PLDE (22) it is important to use methods that preserve the symmetry of the solution and, if appropriate, also its positive definiteness. For example, methods that are able to preserve positive definiteness have been proposed in [25].

\section{SOLUTION OF DPRE}

In this section we address the computation of the periodic stabilizing solution $X_{k}$ of the DPRE (5). Note, however, that any method to solve the DPRE (5) can also be employed to solve the dual equation (10) by solving the DPRE (5) with the replacements:

$$
A_{k} \leftarrow A_{N-k}^{\mathrm{T}}, \quad B_{k} \leftarrow C_{N-k}^{\mathrm{T}}, \quad C_{k} \leftarrow B_{N-k}^{\mathrm{T}}, \quad S_{k} \leftarrow S_{N-k}^{\mathrm{T}}, \quad R_{k} \leftarrow R_{N-k}
$$

From the computed solution $X_{k}$ of the DPRE, the solution of the dual equation is recovered with the replacements:

$$
X_{k} \leftarrow X_{N-k+2}, \quad k=2, \ldots, N
$$




\section{A. VARGA}

\subsection{Existence results}

First, we introduce some basic concepts for discrete-time periodic systems that are suitable for problems with time-varying dimensions [27]. The transition matrix between time moments $i$ and $j$ is defined as $\Phi_{A}(j, i):=A_{j-1} \ldots A_{i+1} A_{i}$, with $\Phi_{A}(i, i):=I_{n_{i}}$. For an $N$-periodic $A_{k}, \Psi_{A}(j):=$ $\Phi_{A}(j+N, j)$ is called the monodromy matrix at time $j$ corresponding to $A_{k}$ and its eigenvalues are called characteristic multipliers at time $j$. The spectrum $\Lambda\left(\Phi_{A}(j+N, j)\right)$ contains at least $n_{j}-\underline{n}$ zero elements, where $\underline{n}:=\min _{k}\left\{n_{k}\right\}$. The rest of $\underline{n}$ eigenvalues are independent of $j$ and form the core characteristic multipliers [28]. We say that $A_{k}$ is a stable periodic matrix if all its characteristic multipliers have magnitudes less than 1.

The notions of reachability/stabilizability and observability/detectability of periodic systems can be defined in a very similar manner to the continuous-time case [27]. A characteristic multiplier $\lambda$ of $A_{k}$ is said to be unreachable at time $\tau$ if $\Psi_{A}^{\mathrm{T}}(\tau) x=\lambda x$ and $x \neq 0$ imply that $B_{j-1}^{\mathrm{T}} \Phi_{A}^{\mathrm{T}}(\tau, j) x=0$, for $j=\tau, \ldots, \tau+N-1$. A characteristic multiplier $\lambda$ of $A_{k}$ is said to be reachable if it is not unreachable. System (6) is said to be stabilizable if all characteristic multipliers $\lambda$ with $|\lambda| \geqslant 1$ are reachable. Similarly, a characteristic multiplier $\lambda$ of $A_{k}$ is said to be unobservable at time $\tau$ if $\Psi_{A}(\tau) x=\lambda x$ and $x \neq 0$ imply that $C_{j} \Phi_{A}(j, \tau) x=0$, for $j=\tau, \ldots, \tau+N-1$. A characteristic multiplier $\lambda$ of $A_{k}$ is said to be observable if it is not unobservable. System (6) is said to be detectable if all characteristic multipliers $\lambda$ with $|\lambda| \geqslant 1$ are observable.

A solution $X_{k}$ of (5) is called a stabilizing solution if the corresponding feedback (8) ensures that $A_{k}+B_{k} F_{k}$ is stable. The following results give necessary and sufficient conditions for the existence of symmetric positive-semidefinite stabilizing solutions for problems with constant dimensions. Since the proofs in [1], based on a standard lifting technique, cover formally the case of timevarying dimensions, these results also apply to this more general case. The following result is the discrete-time counterpart of Theorem 1 [13].

\section{Theorem 4}

The DPRE (5) admits a unique $N$-periodic stabilizing solution $X_{k}=X_{k}^{\mathrm{T}} \geqslant 0$ if and only if the pair $\left(A_{k}, B_{k}\right)$ is stabilizable and the pair $\left(A_{k}, C_{k}\right)$ is detectable.

A less restrictive assumption guarantees the existence of a (possibly non-unique) stabilizing symmetric positive-semidefinite stabilizing solution [1]:

\section{Theorem 5}

The DPRE (5) admits an $N$-periodic stabilizing solution $X_{k}=X_{k}^{\mathrm{T}} \geqslant 0$ if and only if the pair $\left(A_{k}, B_{k}\right)$ is stabilizable and no unit-modulus characteristic multiplier of $A_{k}$ is unobservable.

Note that this theorem is also applicable in the important case $C_{k}=0, k=1, \ldots, N$.

\subsection{Extended periodic $Q Z$ algorithm}

Consider the periodic matrix pairs:

$$
M_{k}=\left[\begin{array}{ccc}
A_{k} & O & B_{k} \\
-Q_{k} & I_{n_{k}} & -S_{k} \\
S_{k}^{\mathrm{T}} & O & R_{k}
\end{array}\right], \quad L_{k}=\left[\begin{array}{ccc}
I_{n_{k+1}} & O & O \\
O & A_{k}^{\mathrm{T}} & O \\
O & -B_{k}^{\mathrm{T}} & O
\end{array}\right]
$$


where $M_{k} \in \mathbb{R}^{\left(n_{k}+n_{k+1}+m_{k}\right) \times\left(2 n_{k}+m_{k}\right)}$ and $L_{k} \in \mathbb{R}^{\left(n_{k}+n_{k+1}+m_{k}\right) \times\left(2 n_{k+1}+m_{k+1}\right)}$. With these pairs, the DPRE (5) together with (8) can be expressed as

$$
M_{k}\left[\begin{array}{c}
I_{n_{k}} \\
X_{k} \\
F_{k}
\end{array}\right]=L_{k}\left[\begin{array}{c}
I_{n_{k+1}} \\
X_{k+1} \\
F_{k+1}
\end{array}\right] \Theta_{k}
$$

for $\Theta_{k}:=A_{k}+B_{k} F_{k} \in \mathbb{R}^{n_{k+1} \times n_{k}}$. This alternative form of the DPRE leads to a straightforward extension of the methods of [9] to compute the stabilizing periodic solution $X_{k}$ :

1. Compute the $N$-periodic matrices $V_{k}$ and $Z_{k}$ such that for $k=1, \ldots, N$

$$
\begin{aligned}
V_{k} M_{k} Z_{k} & =\left[\begin{array}{cc}
H_{k, 11} & H_{k, 12} \\
O & H_{k, 22}
\end{array}\right] \\
V_{k} L_{k} Z_{k+1} & =\left[\begin{array}{cc}
T_{k, 11} & T_{k, 12} \\
O & T_{k, 22}
\end{array}\right]
\end{aligned}
$$

where $H_{k, 11} \in \mathbb{R}^{n_{k+1} \times n_{k}}, T_{k, 11} \in \mathbb{R}^{n_{k+1} \times n_{k+1}}$ is invertible, and all eigenvalues of $\Psi_{T_{11}^{-1} H_{11}}$ (1) are in the unit circle.

2. Partition the rows of $Z_{k}$ to conform with the column partitioning of matrix $M_{k}$ and partition the columns of $Z_{k}$ to conform with the column partitioning of $V_{k} M_{k} Z_{k}$

$$
Z_{k}=\left[\begin{array}{ll}
Z_{k, 11} & Z_{k, 12} \\
Z_{k, 21} & Z_{k, 22} \\
Z_{k, 31} & Z_{k, 32}
\end{array}\right]
$$

If all $Z_{k, 11}$ are invertible, compute $X_{k}=Z_{k, 21} Z_{k, 11}^{-1}$ and $F_{k}=Z_{k, 31} Z_{k, 11}^{-1}$.

Note that in the case of time-varying dimensions, the direct application of the periodic QZ algorithm at Step 1 is not possible. It is shown in what follows how to overcome this difficulty with recently developed algorithms to reduce periodic matrix pairs to orthogonally similar canonical forms.

The computation of $V_{k}$ and $Z_{k}$ at Step 1 can be performed in three main steps. In the first step, a finite-infinite spectral separation is performed by using the recently developed algorithm to compute Kronecker-like forms of periodic pairs [29]. A specialization of this algorithm to perform only the finite-infinite spectral separation is given in Appendix A. By applying this algorithm to the dual periodic pair $\left(\widetilde{M}_{k}, \widetilde{L}_{k}\right)$, where

$$
\tilde{M}_{k}=M_{N-k+1}^{\mathrm{T}}, \quad k=1, \ldots, N, \quad \widetilde{L}_{k}=L_{N-k}^{\mathrm{T}}, \quad k=1, \ldots, N-1, \quad \widetilde{L}_{N}=L_{N}^{\mathrm{T}}
$$

and performing suitable row and column permutations (see [29] for details), orthogonal matrices $V_{k}^{1}$ and $Z_{k}^{1}$ can be determined such that

$$
\begin{aligned}
V_{k}^{1} M_{k} Z_{k}^{1} & =\left[\begin{array}{cc}
M_{k}^{f} & M_{k}^{f, \infty} \\
O & M_{k}^{\infty}
\end{array}\right] \\
V_{k}^{1} L_{k} Z_{k+1}^{1} & =\left[\begin{array}{cc}
L_{k}^{f} & L_{k}^{f, \infty} \\
O & L_{k}^{\infty}
\end{array}\right]
\end{aligned}
$$




\section{A. VARGA}

where $L_{k}^{f}$ and $M_{k}^{\infty}$ are non-singular matrices. The pair $\left(M_{k}^{f}, L_{k}^{f}\right)$ contains the finite characteristic multipliers, whereas the pair $\left(M_{k}^{\infty}, L_{k}^{\infty}\right)$ contains the infinite characteristic multipliers. Note that the finite characteristic multipliers at time $k$ are the eigenvalues of the product $\left(L_{k+N-1}^{f}\right)^{-1} M_{k+N-1}^{f} \ldots\left(L_{k}^{f}\right)^{-1} M_{k}^{f}$ and the nilpotent matrix product $\left(M_{k}^{\infty}\right)^{-1} L_{k}^{\infty} \ldots\left(M_{k+N-1}^{\infty}\right)^{-1}$ $L_{k+N-1}^{\infty}$ characterizes the infinite characteristic multipliers. The problem is not solvable if the above separation cannot be performed because the associated lifted pencil (see Section 3.4) is not regular. Equivalently, the resulting periodic Kronecker-like form contains parts that correspond to a left or right Kronecker structure.

In the second step, orthogonal matrices $V_{k}^{2}$ and $Z_{k}^{2}$ are determined such that

$$
\begin{aligned}
V_{k}^{2} M_{k}^{f} Z_{k}^{2} & =\left[\begin{array}{cc}
M_{k, 11}^{f} & M_{k, 12}^{f} \\
O & M_{k, 22}^{f}
\end{array}\right] \\
V_{k}^{2} L_{k}^{f} Z_{k+1}^{2} & =\left[\begin{array}{cc}
L_{k, 11}^{f} & L_{k, 11}^{f} \\
O & L_{k, 22}^{f}
\end{array}\right]
\end{aligned}
$$

where the pair $\left(M_{k, 22}^{f}, L_{k, 22}^{f}\right)$ has constant dimensions and is in a periodic generalized Hessenberg form [9], $L_{k, 11}^{f} \in \mathbb{R}^{\left(n_{k+1}-\underline{n}\right) \times\left(n_{k+1}-\underline{n}\right)}$ is upper triangular and $M_{k, 11}^{f} \in \mathbb{R}^{\left(n_{k+1}-\underline{n}\right) \times\left(n_{k}-\underline{n}\right)}$ is upper trapezoidal, with $\underline{n}=\min n_{k}$. The pair $\left(M_{k, 11}^{f}, L_{k, 11}^{f}\right)$ has only characteristic multipliers equal to zero. This reduction can be performed by extending the generalized periodic Hessenberg reduction procedures of $[8,9]$ to the case of non-constant dimensions similarly as done in [30] for the periodic Hessenberg form.

Finally, orthogonal matrices $V_{k}^{3}$ and $Z_{k}^{3}$ are computed such that

$$
\begin{gathered}
V_{k}^{3} M_{k, 22}^{f} Z_{k}^{3}=\left[\begin{array}{cc}
M_{k}^{s} & M_{k}^{s, u} \\
O & M_{k}^{u}
\end{array}\right] \\
V_{k}^{3} L_{k, 22}^{f} Z_{k+1}^{3}=\left[\begin{array}{cc}
L_{k}^{s} & L_{k}^{s, u} \\
O & L_{k}^{u}
\end{array}\right]
\end{gathered}
$$

where the pair $\left(M_{k}^{s}, L_{k}^{s}\right)$ has only stable characteristic multipliers, and the pair $\left(M_{k}^{u}, L_{k}^{u}\right)$ has only unstable characteristic multipliers. Because of the symplectic nature of the eigenvalue problem, the non-zero eigenvalues must appear in reciprocal pairs. For this step, the algorithms of $[8,9]$ to compute and reorder the periodic QZ decomposition can be used.

The final transformation matrix $Z_{k}$ is obtained as

$$
Z_{k}=Z_{k}^{1} \operatorname{diag}\left(I, Z_{k}^{2}\right) \operatorname{diag}\left(I, Z_{k}^{3}\right)
$$

To compute $X_{k}$ and $F_{k}$, the accumulation of the left transformations $V_{k}$ is not necessary.

Each computational step of the above algorithm is numerically stable and has a computational complexity of $O\left(N(2 n+m)^{3}\right)$, where $n$ and $m$ are the maximum problem dimensions for $n_{k}$ and $m_{k}$, respectively. 


\section{PERIODIC RICCATI EQUATIONS}

\subsection{Periodic $Q Z$ algorithm with infinite structure deflation}

An approach similar to that proposed for standard systems in [31] can be used to deflate trivial infinite characteristic multipliers first. For this purpose, orthogonal $V_{k}^{1}$ are chosen such that

$$
V_{k}^{1}\left[\begin{array}{c}
B_{k} \\
-S_{k} \\
R_{k}
\end{array}\right]=\left[\begin{array}{c}
O \\
O \\
\bar{R}_{k}
\end{array}\right]
$$

where all $\bar{R}_{k} \in \mathbb{R}^{m_{k} \times m_{k}}$ are non-singular. Note that this is a necessary condition for the existence of a solution of the DPRE. Let us define the reduced periodic pair $\left(\bar{M}_{k}, \bar{L}_{k}\right)$ from

$$
V_{k}^{1} M_{k}=\left[\begin{array}{cc}
\bar{M}_{k} & O \\
* & \bar{R}_{k}
\end{array}\right], \quad V_{k}^{1} L_{k}=\left[\begin{array}{cc}
\bar{L}_{k} & O \\
* & O
\end{array}\right]
$$

where $\bar{M}_{k} \in \mathbb{R}^{\left(n_{k}+n_{k+1}\right) \times 2 n_{k}}, \bar{L}_{k} \in \mathbb{R}^{\left(n_{k}+n_{k+1}\right) \times 2 n_{k+1}}$.

Then the $N$-periodic orthogonal transformation matrices $V_{k}^{2}$ and $Z_{k}^{2}$ can be computed (see Appendix B) such that for $k=1, \ldots, N$ the resulting matrices can be partitioned as follows:

$$
\begin{aligned}
V_{k}^{2} \bar{M}_{k} Z_{k}^{2} & =\left[\begin{array}{ccc}
M_{k}^{0} & * & * \\
O & M_{k}^{c} & * \\
O & O & M_{k}^{\infty}
\end{array}\right] \\
V_{k}^{2} \bar{L}_{k} Z_{k+1}^{2} & =\left[\begin{array}{ccc}
L_{k}^{0} & * & * \\
O & L_{k}^{c} & * \\
O & O & L_{k}^{\infty}
\end{array}\right]
\end{aligned}
$$

where $M_{k}^{0} \in \mathbb{R}^{\left(n_{k+1}-\underline{n}\right) \times\left(n_{k}-\underline{n}\right)}, M_{k}^{c} \in \mathbb{R}^{2 \underline{n} \times 2} \underline{\underline{n}}, M_{k}^{\infty} \in \mathbb{R}^{\left(n_{k}-\underline{n}\right) \times\left(n_{k}-\underline{n}\right)}, L_{k}^{0} \in \mathbb{R}^{\left(n_{k+1}-\underline{n}\right) \times\left(n_{k+1}-\underline{n}\right)}, L_{k}^{c} \in$ $\mathbb{R}^{2 \underline{n} \times 2 \underline{n}}, L_{k}^{\infty} \in \mathbb{R}^{\left(n_{k}-\underline{n}\right) \times\left(n_{k+1}-\underline{n}\right)}$, and $M_{k}^{\infty}$ and $L_{k}^{0}$ are invertible. The periodic pair $\left(M_{k}^{0}, L_{k}^{0}\right)$ contains the trivial zero characteristic values of the pair $\left(\bar{M}_{k}, \bar{L}_{k}\right)$, whereas the periodic pair $\left(M_{k}^{\infty}, L_{k}^{\infty}\right)$ contains the trivial infinite characteristic values. At each time moment, the numbers of zero and infinite characteristic values are equal. As shown in the previous subsection, their number depends, however, on the starting time moment used to define the period.

The periodic pair $\left(M_{k}^{c}, L_{k}^{c}\right)$ contains the $2 \underline{n}$ core characteristic values of the original pair $\left(\bar{M}_{k}, \bar{L}_{k}\right)$ and the characteristic value spectrum is symmetric with respect to the unit circle. We can employ the standard periodic QZ algorithm for constant dimensions $[8,9]$ to separate the stable and unstable characteristic value spectra. We obtain with appropriate orthogonal transformation matrices $V_{k}^{3}$ and $Z_{k}^{3}$

$$
V_{k}^{3} M_{k}^{c} Z_{k}^{3}=\left[\begin{array}{cc}
M_{k}^{s} & * \\
O & M_{k}^{u}
\end{array}\right], \quad V_{k}^{3} L_{k}^{c} Z_{k+1}^{3}=\left[\begin{array}{cc}
L_{k}^{s} & * \\
O & L_{k}^{s}
\end{array}\right]
$$

where $M_{k}^{s} \in \mathbb{R}^{\underline{n} \times \underline{n}}, L_{k}^{s} \in \mathbb{R} \underline{n} \times \underline{n}$ is invertible, and $\Lambda\left(\Psi_{\left(L^{s}\right)^{-1} M^{s}}(1)\right) \subset \mathbb{C}^{-}$. 


\section{A. VARGA}

Finally, let us partition $\bar{Z}_{k}:=Z_{k}^{2} \operatorname{diag}\left(I_{n_{k}-\underline{n}}, Z_{k}^{3}, I_{n_{k}-\underline{n}}\right)$ such that its first $n_{k}$ columns correspond to the stable spectrum (i.e. the trivial zero characteristic values and the stable core characteristic values) of the pair $\left(\bar{M}_{k}, \bar{L}_{k}\right)$

$$
\bar{Z}_{k}=\left[\begin{array}{ll}
\bar{Z}_{k, 11} & \bar{Z}_{k, 12} \\
\bar{Z}_{k, 21} & \bar{Z}_{k, 22}
\end{array}\right]
$$

and $\bar{Z}_{k, 11}$ is square. Provided all $\bar{Z}_{k, 11}$ are invertible, the solution of the DPRE is given by

$$
X_{k}=\bar{Z}_{k, 21} \bar{Z}_{k, 11}^{-1}
$$

The state-feedback matrix $F_{k}$ results from (8).

As for the previous method, all deflation steps as well as the QZ-decomposition step are numerically stable and have a computational complexity of $O\left(N(2 n+m)^{3}\right)$. The main advantage of this second approach is that both in the initial deflation step and in the second separation step no numerical rank decisions are necessary. In the case of problems with repeated characteristic values at infinity (e.g. when solving DPRE for a system with pure delays), the final separation step can be still enhanced by performing first the finite-infinite structure separation using the algorithm of Appendix A. It is well known even in the case of constant systems that this approach allows generally to separate finite-infinite structures more reliably in the presence of multiple infinite eigenvalues than a pure QZ-method-based reordering.

\subsection{Fast algorithm}

This algorithm can be explained by defining the associated lifted pencil to the periodic pair $\left(M_{k}, L_{k}\right)$

$$
H-z T:=\left[\begin{array}{ccccc}
M_{1} & -L_{1} & O & \ldots & O \\
O & M_{2} & -L_{2} & \ldots & O \\
\vdots & \ddots & \ddots & \ddots & \vdots \\
O & & & M_{N-1} & -L_{N-1} \\
-z L_{N} & O & \ldots & O & M_{N}
\end{array}\right]
$$

of order $\mu=\sum_{i=1}^{N}\left(2 n_{i}+m_{i}\right)$. Under the usual assumptions for the existence of the solution of the DPRE, this pencil is regular and has no eigenvalues on the unit circle.

The proposed algorithm implicitly constructs a stable deflating subspace of the pencil (26) by employing structure exploiting reductions similar to that employed in [18] for computing periodic systems' zeros. The method can be seen as an extension of the swapping and collapsing approach proposed in $[10,14]$ of quotient-products (see [15]). The basic reduction is performed as follows.

Consider the $\left(n_{1}+2 n_{2}+n_{3}+m_{1}+m_{2}\right)$ th order orthogonal transformation matrix $U_{1}$ compressing the rows of the matrix

$$
\left[\begin{array}{c}
-L_{1} \\
M_{2}
\end{array}\right] \text { to }\left[\begin{array}{c}
R_{1} \\
O
\end{array}\right]
$$


where $R_{1}$ is a non-singular matrix of order $2 n_{2}+m_{2}$. Applying $U_{1}$ to the first two block rows of $H-z T$ we obtain for the non-zero elements

$$
U_{1}\left[\begin{array}{ccc}
M_{1} & -L_{1} & O \\
O & M_{2} & -L_{2}
\end{array}\right]=\left[\begin{array}{ccc}
\widetilde{M}_{1} & R_{1} & -\widetilde{L}_{1} \\
\widehat{M}_{2} & O & -\widehat{L}_{2}
\end{array}\right]
$$

which defines the new matrices $\widehat{M}_{2}$ and $\widehat{L}_{2}$ with $n_{1}+n_{3}+m_{1}$ rows.

Then construct the $\left(n_{1}+2 n_{i+1}+n_{i+2}+m_{1}+m_{i+1}\right)$ th-order orthogonal transformations $U_{i}$ for $i=2, \ldots, N-1$ such that

$$
U_{i}\left[\begin{array}{ccc}
\widehat{M}_{i} & -\widehat{L}_{i} & O \\
O & M_{i+1} & -L_{i+1}
\end{array}\right]=\left[\begin{array}{ccc}
\tilde{M}_{i} & R_{i} & -\widetilde{L}_{i} \\
\widehat{M}_{i+1} & O & -\widehat{L}_{i+1}
\end{array}\right]
$$

where $R_{i}$ are invertible matrices of order $2 n_{i+1}+m_{i+1}$. This recursively defines the new matrices $\widehat{M}_{i+1}$ and $\widehat{L}_{i+1}$ with $n_{1}+n_{i+2}+m_{1}$ rows.

Applying the transformations $U_{i}$ successively to the $i$ th and $(i+1)$ th block rows of the transformed pencil $H-z T$, the reduced pencil

$$
\bar{H}-z \bar{T}=\left[\begin{array}{c|cccc}
\tilde{M}_{1} & R_{1} & -\widetilde{L}_{1} & O & O \\
\tilde{M}_{2} & O & R_{2} & \ddots & O \\
\vdots & \vdots & \vdots & \ddots & -\widetilde{L}_{N-2} \\
\tilde{M}_{N-1}-z \widetilde{L}_{N-1} & O & O & \cdots & R_{N-1} \\
\hline \widehat{M}_{N}-z \widehat{L}_{N} & O & O & \ldots & O
\end{array}\right]
$$

is obtained, which is orthogonally similar to $H-z T$. Since matrices $R_{i}$ are non-singular, the regular subpencil $\widehat{M}_{N}-z \widehat{L}_{N}$ of order $2 n_{1}+m_{1}$ will contain all finite eigenvalues of the original pencil. To check the regularity of the extended pencil, the reciprocal condition numbers of the upper triangular matrices $R_{i}$ can be cheaply estimated to detect possible rank losses.

To compute the solution $X_{1}$, orthogonals $V_{1}$ and $Z_{1}$ are determined such that

$$
V_{1}\left(\widehat{M}_{N}-z \widehat{L}_{N}\right) Z_{1}=\left[\begin{array}{cc}
H_{11}-z T_{11} & H_{12}-z T_{12} \\
O & H_{22}-z T_{22}
\end{array}\right]
$$

where $H_{11}-z T_{11}$ has only finite and stable eigenvalues. Partition $Z_{1}$ conformably into two block columns and three block rows

$$
Z_{1}=\left[\begin{array}{ll}
Z_{1,11} & Z_{1,12} \\
Z_{1,21} & Z_{1,22} \\
Z_{1,31} & Z_{1,32}
\end{array}\right]
$$

such that $Z_{1,11} \in \mathbb{R}^{n_{1} \times n_{1}}$. Provided $Z_{1,11}$ is invertible, the solution $X_{1}$ and feedback $F_{1}$ can be computed as $X_{1}=Z_{1,21} Z_{1,11}^{-1}$ and $F_{1}=Z_{1,31} Z_{1,11}^{-1}$. The rest of the solution $X_{k}$ and the corresponding feedback gain $F_{k}$ for $k=N, \ldots, 2$ are computed iteratively with (5) and (8). Since the process to iterate directly on Equation (5) is convergent [13], this iteration produces virtually no errors. 


\section{A. VARGA}

To estimate the computational effort of the fast approach, let us assume constant dimensions $n$ and $m$ for matrices $A_{k} \in \mathbb{R}^{n \times n}$ and $B_{k} \in \mathbb{R}^{n \times m}$. The reduction of the pencil $H-z T$ can be done by computing successively $N-1$ QR decompositions of $(4 n+2 m) \times(2 n+m)$ matrices and applying the transformation to two sub-blocks of the same dimensions. The reduction step thus has a computational complexity of $O\left((N-1)(2 n+m)^{3}\right)$. Since the last step, the computation of stable deflating subspace of the reduced pencil $\widehat{M}_{N}-z \widehat{L}_{N}$, has a complexity of $O\left((2 n+m)^{3}\right)$, it follows that the overall computational complexity of the proposed approach corresponds to what is expected for a satisfactory algorithm for periodic systems. Although the periodic QZ-algorithmbased approach has the same computational complexity, the fast approach is substantially more efficient not only because it requires less number of operations but also because it is rich in the so-called level 3 BLAS operations. This justifies the term fast algorithms introduced in [15].

Since the main reduction consists of successive QR-decompositions, it can be shown [32] that the matrices of the computed reduced pencil $\bar{H}-\lambda \bar{T}$ satisfy

$$
\|U X-\bar{X}\|_{2} \leqslant \varepsilon_{M} f(2 n+m)\|X\|_{2}, \quad X=H, T
$$

where $U$ is the matrix of accumulated left orthogonal transformations, $\varepsilon_{M}$ is the relative machine precision, and $f(2 n+m)$ is a quantity of order $2 n+m$. The subsequent computational step is performed using the algorithm of [33] for a preliminary finite-infinite spectrum separation in combination with the deflating subspace method to solve DARE of [31]. Thus, this step is also based exclusively on orthogonal transformations and therefore is numerically stable as well. Overall, it is thus guaranteed that the computed solution is exact for a slightly perturbed extended pencil. It follows that the proposed algorithm to solve the DPRE is numerically backward stable in a certain sense.

Since the structure of the perturbed extended pencil is not preserved in the reduction, we cannot say, however, that the computed zeros are exact for slightly perturbed original data (i.e. the algorithm is not strongly stable). In spite of this weaker type of stability, the proposed algorithm is guaranteed to be numerically reliable, computationally efficient, and straightforward to implement using available standard software.

Regarding the accuracy of the computed solution, it is to be expected that the extended periodic QZ algorithm produces in general more accurate results than the fast algorithm. This claim is supported by recent results on structured condition numbers for invariant subspaces [34], which show that invariant subspaces of block cyclic matrices can be much more sensitive to unstructured perturbations (as those introduced by the fast approach) than to structured perturbations.

\subsection{Application to deadbeat control}

An interesting application of the proposed techniques addresses the case of singular weighting matrices $R_{k}$. Note that this case has not been addressed in any of the existing methods [9-11,14]. The PLQ-optimization-based approach proposed in [17] for deadbeat control can be easily extended to periodic systems. To this end, a PLQ optimization problem with $R_{k}=0, S_{k}=0$, and $Q_{k}=C_{k}^{\mathrm{T}} C_{k}$ is solved, where $C_{k} \in \mathbb{R}^{m_{k} \times n_{k}}$ are chosen such that the periodic system $\left(A_{k}, B_{k}, C_{k}\right)$ has no finite zeros. For standard systems the choice of $C_{k}$ is straightforward and involves the computation of the Kalman controllability form [17]. The same approach can be used for periodic systems by computing the periodic Kalman reachability form $\left(\widetilde{A}_{k}, \widetilde{B}_{k}\right)$ of the periodic pair $\left(A_{k}, B_{k}\right)$ using the algorithm proposed in [35]. For a completely reachable pair $\left(A_{k}, B_{k}\right)$, this algorithm computes 
PERIODIC RICCATI EQUATIONS

orthogonal $N$-periodic transformation matrices $Z_{k}$ such that

$$
\widetilde{A}_{k}=Z_{k+1}^{\mathrm{T}} A_{k} Z_{k}, \quad \widetilde{B}_{k}=Z_{k+1}^{\mathrm{T}} B_{k}
$$

where each matrix $\left[\begin{array}{ll}\widetilde{B}_{k} & \widetilde{A}_{k}\end{array}\right]$ is in a staircase form

$$
\left[\widetilde{B}_{k} \mid \widetilde{A}_{k}\right]=\left[\begin{array}{c|cccc}
A_{k ; 1,0} & A_{k ; 1,1} & A_{k ; 1,2} & \ldots & A_{k ; 1, \ell} \\
O & A_{k ; 2,1} & A_{k ; 2,2} & \ldots & A_{k ; 2, \ell} \\
\vdots & \vdots & \ddots & \ddots & \vdots \\
O & O & O & A_{k ; \ell, \ell-1} & A_{k ; \ell, \ell}
\end{array}\right]
$$

with $A_{k ; i, i} \in \mathbb{R}^{v_{k+1}^{(i)} \times v_{k}^{(i)}}, i=1, \ldots, \ell ; A_{k ; i, i-1} \in \mathbb{R}^{v_{k+1}^{(i)} \times v_{k}^{(i-1)}} ;$ and $\operatorname{rank} A_{k ; i, i-1}=v_{k+1}^{(i)}, i=1, \ldots, \ell$.

$C_{k}$ can be chosen in the form

$$
C_{k}=\operatorname{diag}\left(C_{k, 1}, \ldots, C_{k, \ell-1}, I_{v_{k}^{(\ell)}}\right) Z_{k}^{\mathrm{T}}
$$

where $C_{k, i-1} \in \mathbb{R}^{\left(v_{k}^{(i-1)}-v_{k+1}^{(i)}\right) \times v_{k}^{(i-1)}}$ contains in each line a single non-zero element (e.g. set to 1$)$ in the position corresponding to a linearly dependent column of $A_{k ; i, i-1}$. For instance, in the simple case when all sub-diagonal blocks are invertible (see Example 4 in the following section), $C_{k}$ can be simply chosen as

$$
C_{k}=\left[O \ldots O I_{v_{k}^{(\ell)}}\right] Z_{k}^{\mathrm{T}}
$$

\section{NUMERICAL EXPERIMENTS}

In this section several examples are considered to illustrate the capabilities of the proposed algorithms. For the solution of DPRE computational results are reported only for the fast algorithm for which reliable numerical implementations are available in the recently developed Periodic Systems Toolbox for MATLAB [19]. To assess the accuracy of the results, the residuals (assuming $S_{k}=0$ )

$$
r_{k}=\left\|X_{k}-Q_{k}-A_{k}^{\mathrm{T}} X_{k+1}\left(A_{k}+B_{k} F_{k}\right)\right\|_{F}
$$

can be computed, from which a total residual can be defined as

$$
\text { Residual }=\left(\sum_{j=1}^{N} r_{j}^{2}\right)^{1 / 2}
$$

\section{Example 1}

To illustrate the capabilities of the multiple shooting approach, we consider the numerical solution to the PLDE (12), where we consider

$$
A(t)=\left[\begin{array}{cc}
0 & 1 \\
-10 \cos (t)-1 & -24-10 \sin (t)
\end{array}\right]
$$




\section{A. VARGA}

and we choose $Q(t)$ such that the exact solution of (12) is

$$
X(t)=\left[\begin{array}{cc}
1+\cos (t) & 0 \\
0 & 1+\sin (t)
\end{array}\right]
$$

The period of the problem is $T=2 \pi$. The characteristic multipliers of $A(t)$ are $\mathrm{e}^{-0.046 T}$ and $\mathrm{e}^{-23.95 T}$; thus, the problem is moderately stiff and moderately ill-conditioned.

We computed the unique periodic solution $\bar{X}(t)$ of (12) using sampling periods $\Delta=T / N$, for $N=1,16,64,128,256$. The value of $N=1$ corresponds to the single shooting method. For the discretization of the continuous-time problem and the solution of the resulting discretized periodic Lyapunov equations, tools available in the Periodic Systems Toolbox [19] have been used. In Table I we present accuracy results obtained using three MATLAB ODE solvers: the DormandPrince Runge-Kutta $(4,5)$ code ode45, the non-stiff variable order Adams-Bashforth-Moulton solver ode113, and the numerical differentiation-formulas-based stiff solver ode15s all with both relative and absolute tolerances set to $10^{-8}$. The accuracy of the solution is evaluated as $\left\|X\left(t_{k}\right)-\bar{X}\left(t_{k}\right)\right\|_{2}$ in each point $t_{k}=(k-1) \Delta$, for $k=1, \ldots, N$.

Although the accuracy of the multiple shooting method is always better than that of the single shooting approach, the accuracy gains obtained for the Runge-Kutta method are remarkable. Note that the more accurate multistep methods implemented in ode113 and ode $15 \mathrm{~s}$ achieve their limiting accuracy already for $N=64$ and 16, respectively. We expect that similar conclusions can also be drawn for the solution of PRDEs.

\section{Example 2}

This is example 2 of [9] and has a singular monodromy matrix. We solved for this problem the deadbeat control problem by choosing $R_{1}=R_{2}=R_{3}=0$ and

$$
Q_{1}=\frac{1}{66}\left[\begin{array}{ccc}
64 & -8 & 8 \\
-8 & 1 & -1 \\
8 & -1 & 1
\end{array}\right], \quad Q_{2}=\frac{1}{227}\left[\begin{array}{ccc}
1 & -1 & -15 \\
-1 & 1 & 15 \\
-15 & 15 & 225
\end{array}\right], \quad Q_{3}=\frac{1}{10}\left[\begin{array}{ccc}
1 & 0 & -3 \\
0 & 0 & 0 \\
-3 & 0 & 9
\end{array}\right]
$$

The resulting exact solution is

$$
F_{1}=\left[\begin{array}{lll}
30 & -20 & 2
\end{array}\right], \quad F_{2}=\frac{1}{39}\left[\begin{array}{lll}
-118 & 59 & -74
\end{array}\right], \quad F_{3}=\left[\begin{array}{lll}
8 & -48 & 0
\end{array}\right]
$$

The computed floating-point solution had a total residual of $2.53 \times 10^{-10}$.

Table I. Accuracy results for $\max \left\|X\left(t_{k}\right)-\bar{X}\left(t_{k}\right)\right\|_{2}$.

\begin{tabular}{lccc}
\hline$N$ & ode45 & ode113 & ode15s \\
\hline 1 & $1.6 \times 10^{-4}$ & $1.9 \times 10^{-8}$ & $2.4 \times 10^{-7}$ \\
16 & $1.4 \times 10^{-6}$ & $8.3 \times 10^{-9}$ & $2.1 \times 10^{-8}$ \\
64 & $1.1 \times 10^{-7}$ & $5.6 \times 10^{-9}$ & $5.4 \times 10^{-8}$ \\
128 & $9.0 \times 10^{-9}$ & $9.1 \times 10^{-9}$ & $1.1 \times 10^{-7}$ \\
256 & $1.1 \times 10^{-9}$ & $8.3 \times 10^{-9}$ & $8.9 \times 10^{-8}$ \\
\hline
\end{tabular}


PERIODIC RICCATI EQUATIONS

Example 3

This is an example with time-varying dimensions, defined by the 3-periodic matrices

$$
\begin{aligned}
& A_{1}=\left[\begin{array}{ccc}
-3 & 2 & 9 \\
0 & 0 & -4
\end{array}\right], \quad A_{2}=\left[\begin{array}{ll}
6 & -3 \\
4 & -2
\end{array}\right], \quad A_{3}=\left[\begin{array}{cc}
2 & -3 \\
4 & -15 \\
-2 & 9
\end{array}\right] \\
& B_{1}=\left[\begin{array}{l}
1 \\
1
\end{array}\right], \quad B_{2}=\left[\begin{array}{l}
0 \\
1
\end{array}\right], \quad B_{3}=\left[\begin{array}{l}
0 \\
1 \\
1
\end{array}\right]
\end{aligned}
$$

For this problem the deadbeat control problem has been solved by choosing $R_{1}=R_{2}=R_{3}=0$ and

$$
Q_{1}=\left[\begin{array}{ccc}
1 & 0 & 0 \\
0 & \frac{1}{2} & -\frac{1}{2} \\
0 & -\frac{1}{2} & \frac{1}{2}
\end{array}\right], \quad Q_{2}=\left[\begin{array}{cc}
\frac{1}{2} & -\frac{1}{2} \\
-\frac{1}{2} & \frac{1}{2}
\end{array}\right], \quad Q_{3}=\left[\begin{array}{ll}
1 & 0 \\
0 & 0
\end{array}\right]
$$

The resulting total residual of the computed solution is $2.1 \times 10^{-12}$.

For reference purposes, the exact solution of the DPRE and the corresponding periodic deadbeat state-feedback are given as

$$
\begin{aligned}
& X_{1}=\left[\begin{array}{ccc}
\frac{11}{2} & -3 & -\frac{39}{2} \\
-3 & \frac{5}{2} & \frac{25}{2} \\
-\frac{39}{2} & \frac{25}{2} & 85
\end{array}\right], \quad X_{2}=\left[\begin{array}{cc}
\frac{2003}{22} & -\frac{1007}{22} \\
-\frac{1007}{22} & \frac{509}{22}
\end{array}\right] \quad X_{3}=\left[\begin{array}{cc}
23 & -78 \\
-78 & 297
\end{array}\right] \\
& F_{1}=\left[\begin{array}{lll}
6 & -4 & -22
\end{array}\right], \quad F_{2}=\left[\begin{array}{ll}
-\frac{80}{33} & \frac{40}{33}
\end{array}\right], \quad F_{3}=\left[\begin{array}{ll}
\frac{8}{5} & -\frac{32}{5}
\end{array}\right]
\end{aligned}
$$

\section{Example 4}

This example has been used in [36] to design periodic output feedback controllers for periodic systems with relatively large periods. The discrete-time periodic system originates from a continuous-time periodic model of a spacecraft pointing and attitude system described in [37]. This system has state and input dimensions of $n=4, m=1$, respectively, and a period of $T=6073.8 \mathrm{~s}$. The discretized system for different sampling periods $T / N$ has been used in [36] to design periodic output feedback controllers for this system. The matrices of the discrete-time periodic system can be computed explicitly for arbitrary values of $N$.

The deadbeat control problem has been solved for different sampling times by choosing $R_{k}=0$, $S_{k}=0$, and $Q_{k}=C_{k}^{\mathrm{T}} C_{k}$ with $C_{k}=\left[\begin{array}{llll}0 & 0 & 0 & 1\end{array}\right] Z_{k}^{\mathrm{T}}$, where $Z_{k}$ is the $N$-periodic orthogonal transformation matrix that brings the periodic pair $\left(A_{k}, B_{k}\right)$ into the periodic Kalman reachability form $\left(\widetilde{A}_{k}, \widetilde{B}_{k}\right)$. Note that in this form each $\widetilde{B}_{k}$ has only the $(1,1)$ entry non-zero and each $\widetilde{A}_{k}$ is an upper Hessenberg matrix. 


\section{A. VARGA}

Table II. Computational results for satellite deadbeat control.

\begin{tabular}{lcccc}
\hline$N$ & 40 & 120 & 360 & 600 \\
\hline Residual & $1.0 \times 10^{-11}$ & $7.7 \times 10^{-14}$ & $8.4 \times 10^{-12}$ & $2.4 \times 10^{-11}$ \\
ITER & 2 & 2 & 2 & 6 \\
STDEG & $2 \times 10^{-155}$ & $5 \times 10^{-324}$ & $1 \times 10^{-322}$ & $2 \times 10^{-322}$ \\
\hline
\end{tabular}

In Table II we present the following results obtained for different values of $N$ : the total residual (28), ITER - the number of iterations performed on DPRE (5) to achieve the limiting accuracy, and STDEG-the achieved closed-loop stability degree (i.e. the maximum modulus of closed-loop characteristic multipliers). Note that $N=600$ corresponds to a typical sampling period of about $10 \mathrm{~s}$ used to control satellites on low Earth orbits.

\section{CONCLUDING REMARKS}

The proposed algorithms to solve PRDEs, PLDEs, and DPREs are completely general, numerically reliable, and computationally efficient. To solve the PRDE and PLDE, we proposed a multiple shooting-type approach, which computes the periodic solutions in an arbitrary number of equidistant time instants within one period, by employing a suitable discretization of the continuous-time problems. In contrast to traditionally used single shooting periodic generator methods, the multiple shooting method has the advantage of successfully tackling problems with large periods and/or unstable dynamics. The proposed methods to solve DPREs, in contrast to existing methods, are able to address problems with time-varying dimensions and/or with singular weighting matrices. The new methods to solve PRDEs and DPREs can also be used to solve the more general periodic Riccati equations, as those arising in periodic $\mathscr{H}_{\infty}$-synthesis problems [38-40].

For the solution of PLDEs using the multiple shooting approach and the solution of DPREs using the fast algorithm, robust numerical implementations are available in the Periodic Systems Toolbox [19]. The codes for solving DPREs underlie user-friendly software to solve PLQ control and filtering-related problems. This software used in conjunction with software tools to compute periodic Kalman forms allows one to solve periodic deadbeat control problems in the most general setting (i.e. for controllable systems with time-varying dimensions).

\section{APPENDIX A}

We present a special version of the general algorithm proposed in [29] to compute periodic Kronecker-like forms. The resulting algorithm is intended to efficiently perform the finite-infinite spectral separation of the associated lifted regular pencil $P(z):=H-z T$ defined in (26) by manipulating exclusively the given periodic pair $\left(M_{k}, L_{k}\right)$, where $M_{k} \in \mathbb{R}^{\mu_{k} \times v_{k}}$ and $L_{k} \in \mathbb{R}^{\mu_{k} \times v_{k+1}}$. The resulting reduced form of this pair is the required one in the proposed extended periodic QZ algorithm to solve DPRE.

For $N=1$, this algorithm corresponds to the finite-infinite structure separation procedure used to determine the Kronecker structure of a linear pencil [41]. In the case of multiple infinite 
eigenvalues, this approach is the numerically reliable alternative to the reordering approach via the QZ algorithm.

The main computation is to determine orthogonal periodic transformation matrices $Q_{k}$ and $Z_{k}$ such that

$$
Q_{k} M_{k} Z_{k}=\left[\begin{array}{cc}
M_{k}^{\infty} & * \\
O & M_{k}^{f}
\end{array}\right], \quad Q_{k} L_{k} Z_{k+1}=\left[\begin{array}{cc}
L_{k}^{\infty} & * \\
O & L_{k}^{f}
\end{array}\right]
$$

where

(a) $M_{k}^{\infty}$ is invertible and the product $\left(M_{k}^{\infty}\right)^{-1} L_{k}^{\infty} \ldots\left(M_{k+N-1}^{\infty}\right)^{-1} L_{k+N-1}^{\infty}$ is nilpotent and

(b) $L_{k}^{f}$ is non-singular.

Using the above orthogonal transformations to reduce the periodic pair $\left(M_{k}, L_{k}\right)$ to the form in (A1) is equivalent to computing $\widetilde{P}(z)=\mathscr{Q} P(z) \mathscr{Z}$, where $\mathscr{Q}=\operatorname{diag}\left(Q_{1}, Q_{2}, \ldots, Q_{N}\right)$ and $\mathscr{Z}=$ $\operatorname{diag}\left(Z_{1}, Z_{2}, \ldots, Z_{N}\right)$. Since $\widetilde{P}(z)$ has the same cyclic structure as $P(z)$, by using appropriate permutation matrices $\Pi_{1}$ and $\Pi_{2}$ we can reorder its blocks such that

$$
\Pi_{1} \widetilde{P}(z) \Pi_{2}=\left[\begin{array}{cc}
P^{\infty}(z) & * \\
O & P^{f}(z)
\end{array}\right]
$$

with each non-zero block having the same cyclic structure as $P(z)$ in (26). For example, the diagonal blocks have the form

$$
P^{x}(z)=\left[\begin{array}{ccccc}
M_{1}^{x} & -L_{1}^{x} & O & \cdots & O \\
O & M_{2}^{x} & -L_{2}^{x} & \cdots & O \\
\vdots & \ddots & \ddots & \ddots & \vdots \\
O & & & M_{N-1}^{x} & -L_{N-1}^{x} \\
-z L_{N}^{x} & O & \ldots & O & M_{N}^{x}
\end{array}\right]
$$

where the upper index $x$ stands for $\infty$ or $f$. The reduction algorithm that we describe in this appendix guarantees the above properties (a) and (b) of the reduced matrices. It follows that pencils $P^{\infty}(z)$ and $P^{f}(z)$ have only infinite and finite zeros, respectively [29]. Note that the simple infinite eigenvalues of $P^{\infty}(z)$ and $P^{f}(z)$ introduced by lifting play no role in counting zeros (the multiplicities of infinite eigenvalues are excess one to the multiplicities of infinite zeros).

The proposed algorithm has two main steps, which we discuss subsequently.

\section{A.1. Preliminary compression}

In the first step we reduce the problem to an equivalent one, but with a special structure of matrices. Let $Q_{k}^{(1)}$ and $Z_{k}^{(1)}$ be orthogonal periodic matrices such that

$$
M_{k}^{(1)}:=Q_{k}^{(1)} M_{k} Z_{k}^{(1)}=\left[\begin{array}{cc}
B_{k} & A_{k} \\
D_{k} & C_{k}
\end{array}\right], \quad L_{k}^{(1)}:=Q_{k}^{(1)} L_{k} Z_{k+1}^{(1)}=\left[\begin{array}{cc}
O & E_{k} \\
O & O
\end{array}\right]
$$




\section{A. VARGA}

where for $k=1, \ldots, N, E_{k} \in \mathbb{R}^{n_{k+1} \times n_{k+1}}$ is invertible, $A_{k} \in \mathbb{R}^{n_{k+1} \times n_{k}}, B_{k} \in \mathbb{R}^{n_{k+1} \times m_{k}}, C_{k} \in \mathbb{R}^{p_{k} \times n_{k}}$, $D_{k} \in \mathbb{R}^{p_{k} \times m_{k}}$, with $m_{k}:=v_{k}-n_{k}$ and $p_{k}:=\mu_{k}-n_{k+1}$. Owing to the regularity of the lifted pencil, it follows that

$$
\left[\begin{array}{c}
B_{k} \\
D_{k}
\end{array}\right]
$$

must have a full column rank and $\left[D_{k} C_{k}\right]$ must have a full row rank.

The compression of each $L_{k}$ to a non-singular $E_{k}$ can be done by computing a full orthogonal decomposition $Q_{k}^{(1)} L_{k} V_{k+1}=\operatorname{diag}\left(E_{k}, O\right)$ using either the singular-value decomposition or a rankrevealing QR-decomposition followed by an RQ-decomposition. Finally, the form in (A3) is obtained by applying column permutations with an appropriate permutation matrix $\Pi_{k}$. For both rank determination techniques, we can arrange that each resulting $E_{k}$ is upper triangular. Define $Z_{k}^{(1)}=V_{k} \Pi_{k}$.

\section{A.2. Basic reduction}

The goal of this reduction step is to separate the infinite $(\infty)$ and finite $(f)$ zero structures of the reduced lifted pencil $P^{(1)}(z)$, which corresponds to the reduced periodic pair $\left(M_{k}^{(1)}, L_{k}^{(1)}\right)$. In this step we compute orthogonal periodic matrices $Q_{k}^{(2)}$ and $Z_{k}^{(2)}$ such that

$$
M_{k}^{(2)}:=Q_{k}^{(2)} M_{k}^{(1)} Z_{k}^{(2)}=\left[\begin{array}{cc}
M_{k}^{\infty} & * \\
O & M_{k}^{f}
\end{array}\right], \quad T_{k}^{(2)}:=Q_{k}^{(2)} L_{k}^{(1)} Z_{k+1}^{(2)}=\left[\begin{array}{cc}
L_{k}^{\infty} & * \\
O & L_{k}^{f}
\end{array}\right]
$$

where for $k=1, \ldots, N, M_{k}^{\infty} \in \mathbb{R}^{n_{k}^{\infty} \times n_{k}^{\infty}}$ is in a staircase form (see (A5)) with invertible matrices on its main diagonal, $L_{k}^{\infty} \in \mathbb{R}^{n_{k}^{\infty} \times n_{k+1}^{\infty}}$ has the non-zero trailing part of full column rank (see (A6)), $M_{k}^{f} \in \mathbb{R}^{n_{k+1}^{f} \times n_{k}^{f}}$, and $L_{k}^{f} \in \mathbb{R}^{n_{k+1}^{f} \times n_{k+1}^{f}}$ is invertible. The following basic reduction algorithm can be used to identify and separate the finite and infinite structures of a compressed periodic pair:

\section{Algorithm for FINITE-INFINITE separation.}

For $k=1, \ldots, N$, set $n_{k}^{\infty}=0, n_{k}^{f}=n_{k}, \rho_{k}^{(0)}=m_{k}$; set $Q_{k}^{(2)}=I_{\mu_{k}}, Z_{k}^{(2)}=I_{v_{k}}$.

\section{step i}

1. For each $k=1, \ldots, N$, compute (e.g. by performing the QR-decomposition with column pivoting on $D_{k}$ ) an orthogonal matrix $W_{k}$ and a permutation matrix $\Pi_{k}$ such that

$$
\left[\begin{array}{c:c|c}
B_{k, 1} & B_{k, 2} & A_{k} \\
\hline D_{k, 1} & D_{k, 2} & C_{k, 1} \\
\hdashline O & O & C_{k, 2}
\end{array}\right]:=\operatorname{diag}\left(I_{n_{k+1}}, W_{k}\right)\left[\begin{array}{c|c}
B_{k} & A_{k} \\
\hline D_{k} & C_{k}
\end{array}\right] \operatorname{diag}\left(\Pi_{k}, I_{n_{k}}\right)
$$

where $D_{k, 1} \in \mathbb{R}^{\tau_{k}^{(i)} \times \tau_{k}^{(i)}}$ is invertible and upper triangular.

2. For each $k=1, \ldots, N$, compress the rows of

$$
\left[\begin{array}{c}
B_{k, 1} \\
D_{k, 1}
\end{array}\right]
$$


with orthogonal $X_{k}$ such that

$$
\left[\begin{array}{c:c|c}
B_{k, 11} & B_{k, 12} & A_{k, 1} \\
O & B_{k, 22} & A_{k, 2}
\end{array}\right]:=X_{k}\left[\begin{array}{c:c|c}
B_{k, 1} & B_{k, 2} & A_{k} \\
\hline D_{k, 1} & D_{k, 2} & C_{k, 1}
\end{array}\right], \quad\left[\begin{array}{l}
E_{k, 1} \\
\overline{E_{k, 2}}
\end{array}\right]:=X_{k}\left[\begin{array}{c}
E_{k} \\
\bar{O}
\end{array}\right]
$$

where $B_{k, 11} \in \mathbb{R}^{\tau_{k}^{(i)} \times \tau_{k}^{(i)}}$ has a full row rank and is upper triangular and $E_{k, 2} \in \mathbb{R}^{n_{k+1} \times n_{k+1}}$ is invertible and upper triangular (see [42]).

3. For $k=1, \ldots, N$, determine orthogonal $U_{k}$ to compress the rows of $B_{k, 22}$ to an invertible upper triangular matrix such that

$$
U_{k} B_{k, 22}=\left[\begin{array}{c}
\widetilde{B}_{k, 22} \\
O
\end{array}\right]
$$

where $\widetilde{B}_{k, 22} \in \mathbb{R}^{\rho_{k+1}^{(i)} \times\left(\rho_{k}^{(i-1)}-\tau_{k}^{(i)}\right)}$ is invertible, and $\rho_{k+1}^{(i)}:=\rho_{k}^{(i-1)}-\tau_{k}^{(i)}$; compute orthogonal $V_{k+1}$ such that $U_{k} E_{k, 2} V_{k+1}$ is upper triangular.

4. For $k=1, \ldots, N$, form the transformation matrices

$$
\begin{aligned}
& \widetilde{Q}_{k}=\operatorname{diag}\left(I_{\tau_{k}^{(i)}}, U_{k}, I_{p_{k}-\tau_{k}^{(i)}}\right) \operatorname{diag}\left(X_{k}, I_{p_{k}-\tau_{k}^{(i)}}\right) \operatorname{diag}\left(I_{n_{k+1}}, W_{k}\right) \\
& \widetilde{Z}_{k}=\operatorname{diag}\left(\Pi_{k}, I_{n_{k}}\right) \operatorname{diag}\left(I_{m_{k}}, V_{k}\right)
\end{aligned}
$$

and transform the submatrices and partition them as

$$
\begin{aligned}
& \begin{aligned}
\tau_{k}^{(i)} \\
\rho_{k+1}^{(i)} \\
n_{k+1}-\rho_{k+1}^{(i)} \\
v p_{k}-\tau_{k}^{(i)}
\end{aligned}\left[\begin{array}{c:c|c|c}
B_{k, 11} & B_{k, 12} & A_{k, 11} & A_{k, 12} \\
\hdashline O & \widetilde{B}_{k, 22} & A_{k, 21} & A_{k, 22} \\
\hdashline O & O & A_{k, 31} & A_{k, 32} \\
\hdashline O & O & C_{k, 21} & C_{k, 22}
\end{array}\right]:=\widetilde{Q}_{k}\left[\begin{array}{c|c}
B_{k} & A_{k} \\
D_{k} & C_{k}
\end{array}\right] \widetilde{Z}_{k}
\end{aligned}
$$

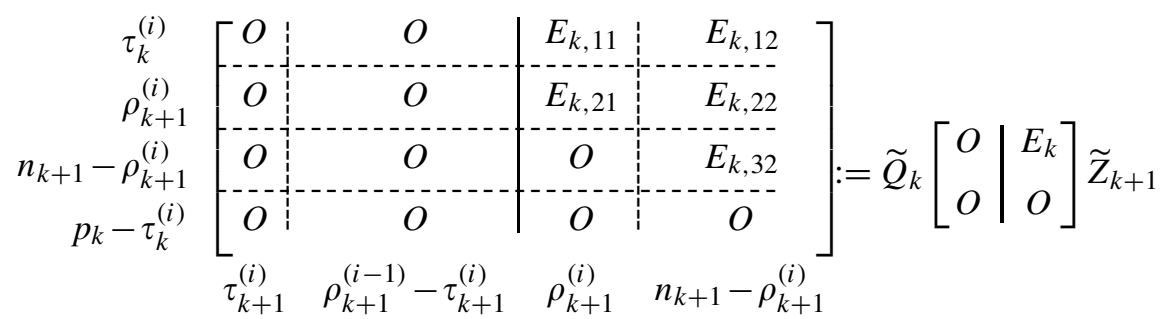

where $B_{k, 11}$ and $\widetilde{B}_{k, 22}$ are invertible and upper triangular, and $E_{k, 21}$ and $E_{k, 32}$ are invertible and upper triangular.

5. For $k=1, \ldots, N$, update $A_{k}:=A_{k, 32}, E_{k}:=E_{k, 32}, B_{k}:=A_{k, 31}, C_{k}:=C_{k, 22}, D_{k}:=C_{k, 21}$.

6. For $k=1, \ldots, N, Q_{k}^{(2)}:=\operatorname{diag}\left(I_{n_{k}^{\infty}}, \widetilde{Q}_{k}\right) Q_{k}^{(2)}, Z_{k}^{(2)}:=Z_{k}^{(2)} \operatorname{diag}\left(I_{n_{k}^{\infty}}, \widetilde{Z}_{k}\right)$.

7. For $k=1, \ldots, N$, update $n_{k}^{\infty}:=n_{k}^{\infty}+\rho_{k}^{(i-1)}, n_{k}^{f}:=n_{k}^{f}-\rho_{k}^{(i)}, p_{k}:=p_{k}-\tau_{k}^{(i)}$. 


\section{A. VARGA}

8. If $\rho_{k}^{(i)}=0$ for $k=1, \ldots, K$, then go to exit

9. $i:=i+1$ go to step $\mathbf{i}$;

exit. Compute $M_{k}^{(2)}:=Q_{k}^{(2)} M_{k}^{(1)} Z_{k}^{(2)}, L_{k}^{(2)}:=Q_{k}^{(2)} L_{k}^{(1)} Z_{k+1}^{(2)}$ and partition them according to (A4).

The computation stops when all $B_{k}$ and $D_{k}$ have null number of columns. The resulting finite periodic pair is $\left(M_{k}^{f}, L_{k}^{f}\right):=\left(A_{k}, E_{k}\right)$, with $M_{k}^{f} \in \mathbb{R}^{n_{k+1}^{f} \times n_{k}^{f}}$, and $L_{k}^{f} \in \mathbb{R}^{n_{k+1}^{f} \times n_{k+1}^{f}}$ invertible and upper triangular. Since the associated lifted pencil has full rank for almost all finite values of $z$, the pair $\left(M_{k}^{f}, L_{k}^{f}\right)$ can have only finite eigenvalues. These are the eigenvalues of $\Psi_{\left(L^{f}\right)^{-1} M^{f}}(k)$.

The compression at Step 2 can be done by performing a QR-decomposition of

$$
\left[\begin{array}{l}
B_{k, 1} \\
D_{k, 1}
\end{array}\right]
$$

which exploits the upper triangular shape of $D_{k, 1}$. This can be achieved by employing sequences of Givens transformations to zero successive elements under the diagonal of $B_{k, 1}$. By starting from below (i.e. zeroing first the diagonal element of $D_{k, 1}$ ) the upper triangular structure of $E_{k, 2}$ is automatically achieved. For details see [42].

The compression at Step 3 of $B_{k, 22}$ to a full row rank matrix can be done simultaneously with maintaining $E_{k, 2}$ upper triangular. This compression technique represents the main computational step in determining the periodic controllability staircase form of periodic descriptor systems (see [35] for more details).

At the end of this algorithm we obtain globally the reduced matrices $M_{k}^{(2)}$ and $L_{k}^{(2)}$ in the form of (A4), where the periodic pair $\left(M_{k}^{\infty}, L_{k}^{\infty}\right)$ is in the following staircase form:

$$
\begin{aligned}
& M_{k}^{\infty}=\left[\begin{array}{ccccc}
M_{k ; 1,1}^{\infty} & M_{k ; 1,2}^{\infty} & \ldots & M_{k ; 1, \ell-1}^{\infty} & M_{k ; 1, \ell}^{\infty} \\
O & M_{k ; 2,2}^{\infty} & \ldots & M_{k ; 2, \ell-1}^{\infty} & M_{k ; 2, \ell}^{\infty} \\
\vdots & \vdots & \ddots & \vdots & \vdots \\
O & O & \ldots & M_{k ; \ell-1, \ell-1}^{\infty} & M_{k ; \ell-1, \ell}^{\infty} \\
O & O & \ldots & O & M_{k ; \ell, \ell}^{\infty}
\end{array}\right] \\
& L_{k}^{\infty}=\left[\begin{array}{ccccc}
O & L_{k ; 1,2}^{\infty} & \ldots & L_{k ; 1, \ell-1}^{\infty} & L_{k ; 1, \ell}^{\infty} \\
O & O & \ldots & L_{k ; 2, \ell-1}^{\infty} & L_{k ; 2, \ell}^{\infty} \\
\vdots & \vdots & \ddots & \vdots & \vdots \\
O & O & \ldots & O & L_{k ; \ell-1, \ell}^{\infty} \\
O & O & \ldots & O & O
\end{array}\right]
\end{aligned}
$$

where $\ell$ is the number of steps performed by the algorithm, $M_{k ; i, i}^{\infty} \in \mathbb{R}^{\left(\rho_{k+1}^{(i)}+\tau_{k}^{(i)}\right) \times \rho_{k}^{(i-1)}}$ is invertible

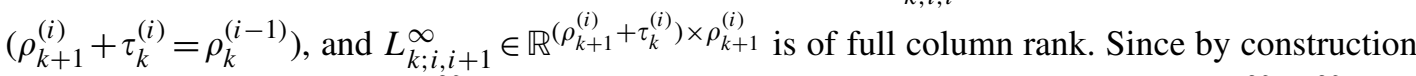
the associated lifted pencil $P^{\infty}(z)$ is invertible for all finite values of $z$, the pair $\left(M_{k}^{\infty}, L_{k}^{\infty}\right)$ has only an infinite structure.

The final transformation matrices are $Q_{k}=Q_{k}^{(2)} Q_{k}^{(1)}$ and $Z_{k}=Z_{k}^{(1)} Z_{k}^{(2)}$. 


\section{PERIODIC RICCATI EQUATIONS}

\section{APPENDIX B}

Here, we propose an algorithm to reduce a periodic pair $\left(M_{k}, L_{k}\right)$, where $M_{k} \in \mathbb{R}^{\left(n_{k}+n_{k+1}\right) \times 2 n_{k}}$, $L_{k} \in \mathbb{R}^{\left(n_{k}+n_{k+1}\right) \times 2 n_{k+1}}$, to a compressed form, where the parts corresponding to trivial characteristic values in the origin and at infinity, originated from the presence of time-varying dimensions, are separated. Let us assume that $n_{1}=\underline{n}:=\min n_{k}$. The following procedure determines orthogonal periodic transformation matrices $Q_{k}$ and $Z_{k}$ to achieve this separation using a sequence of QRand RQ-decompositions:

Algorithm for separation of trivial zero and infinite spectra.

Set $Z_{1}=I_{n_{1}}$ and compute orthogonal $Q_{N}$ (e.g. using the QR-decomposition) such that

$$
T_{N}=Q_{N} L_{N}=\left[\begin{array}{c}
\widetilde{L}_{N, 1} \\
O
\end{array}\right]
$$

where $\widetilde{L}_{N, 1}$ is square and upper triangular.

For $k=N, N-1, \ldots, 2$

1. Compute orthogonal $Z_{k}$ (e.g. using the RQ-decomposition) such that

$$
H_{k}:=Q_{k} M_{k} Z_{k}= \begin{cases}{\left[O \tilde{M}_{k, 2}\right.} & \text { if } n_{k}>n_{k+1} \\
{\left[\begin{array}{c}
\widetilde{M}_{k, 1} \\
\tilde{M}_{k, 2}
\end{array}\right]} & \text { if } n_{k} \leqslant n_{k+1}\end{cases}
$$

where $\widetilde{M}_{k, 2}$ is square and upper triangular.

2. Compute orthogonal $Q_{k-1}$ (e.g. using the QR-decomposition) such that

$$
T_{k-1}=Q_{k-1} L_{k-1} Z_{k}= \begin{cases}{\left[\begin{array}{cc}
\widetilde{L}_{k-1,1} & \widetilde{L}_{k-1,2}
\end{array}\right]} & \text { if } n_{k-1} \leqslant n_{k} \\
{\left[\begin{array}{c}
\widetilde{L}_{k-1,1} \\
O
\end{array}\right]} & \text { if } n_{k-1}>n_{k}\end{cases}
$$

where $\widetilde{L}_{k-1,1}$ is square and upper triangular.

Compute $H_{1}=Q_{1} M_{1}$.

The resulting matrices $H_{k}$ and $T_{k}$ can be partitioned as follows:

$$
\begin{gathered}
H_{k}:=Q_{k} M_{k} Z_{k}=\left[\begin{array}{ccc}
H_{k, 11} & H_{k, 12} & H_{k, 13} \\
O & H_{k, 22} & H_{k, 23} \\
O & O & H_{k, 33}
\end{array}\right] \\
T_{k}:=Q_{k} L_{k} Z_{k+1}=\left[\begin{array}{ccc}
T_{k, 11} & T_{k, 12} & T_{k, 13} \\
O & T_{k, 22} & T_{k, 23} \\
O & O & T_{k, 33}
\end{array}\right]
\end{gathered}
$$

where $H_{k, 11} \in \mathbb{R}^{\left(n_{k+1}-\underline{n}\right) \times\left(n_{k}-\underline{n}\right)}, H_{k, 22} \in \mathbb{R}^{2 \underline{n} \times 2 \underline{n}}, H_{k, 33} \in \mathbb{R}^{\left(n_{k}-\underline{n}\right) \times\left(n_{k}-\underline{n}\right)}, T_{k, 11} \in \mathbb{R}^{\left(n_{k+1}-\underline{n}\right) \times\left(n_{k+1}-\underline{n}\right)}$, $T_{k, 22} \in \mathbb{R}^{2 \underline{n} \times 2 \underline{n}}$, and $T_{k, 33} \in \mathbb{R}^{\left(n_{k}-\underline{n}\right) \times\left(n_{k+1}-\underline{n}\right)}$. Because the lifted pencil associated with the pair 


\section{A. VARGA}

$\left(M_{k}, L_{k}\right)$ is regular, it follows immediately that the upper triangular periodic matrices $H_{k, 33}$ and $T_{k, 11}$ are invertible. The periodic pair $\left(H_{k, 11}, T_{k, 11}\right)$ contains the trivial zero characteristic values of the original pair $\left(M_{k}, L_{k}\right)$, whereas the periodic pair $\left(H_{k, 33}, T_{k, 33}\right)$ contains the trivial infinite characteristic values. The numbers of zero and infinite characteristic values are equal but depend on the starting time moment used to define the period. The periodic pair $\left(H_{k, 22}, T_{k, 22}\right)$ contains the $2 \underline{n}$ core characteristic values of the original pair $\left(M_{k}, L_{k}\right)$. A more refined version of the above algorithm can be devised such that the periodic pair $\left(H_{k, 22}, T_{k, 22}\right)$ results in a generalized periodic Hessenberg form (see $[8,9])$.

\section{ACKNOWLEDGEMENTS}

This work was partially supported by the Swedish Strategic Research Foundation Grant 'Matrix Pencil Computations in Computer-Aided Control System Design: Theory, Algorithms and Software Tools.' The author thanks the anonymous reviewers for their very detailed and careful review, which substantially improved the presentation of the results.

\section{REFERENCES}

1. Bittanti S. The periodic Riccati equation. In The Riccati Equation, Bittanti S, Laub AJ, Willems JC (eds). Springer: Berlin, 1991; 127-162.

2. Colaneri P. Periodic control systems: theoretical aspects. Proceedings of IFAC Workshop on Periodic Systems, Yokohama, Japan, 2005.

3. Brockett RW. Finite Dimensional Linear Systems. Wiley: New York, 1970.

4. De Nicolao G, Strada S. On the use of reachability Gramians for the stabilization of linear periodic systems. Automatica 1997; 33:729-732.

5. Varga A. On solving periodic differential matrix equations with applications to periodic system norms computation. Proceedings of CDC'05, Seville, Spain, 2005.

6. Varga A. On solving discrete-time periodic Riccati equations. Proceedings of IFAC 2005 World Congress, Prague, Czech Republic, 2005.

7. Hench JJ, Kenney CS, Laub AJ. Methods for the numerical integration of Hamiltonian systems. Circuits, Systems and Signal Processing 1994; 13:695-732.

8. Bojanczyk AW, Golub G, Van Dooren P. The periodic Schur decomposition. Algorithms and applications. In Proceedings SPIE Conference, San Diego, vol. 1770, Luk FT (ed.), July 1992; 31-42.

9. Hench JJ, Laub AJ. Numerical solution of the discrete-time periodic Riccati equation. IEEE Transactions on Automatic Control 1994; 39:1197-1210.

10. Benner P, Byers R, Mayo R, Quintana-Orti ES, Hernandez V. Parallel algorithms for LQ optimal control of discrete-time periodic linear systems. Journal of Parallel and Distributed Computing 2002; 62:306-325.

11. Chu EKW, Fan HY, Lin WW, Wang CS. Structure-preserving algorithm for periodic discrete-time algebraic Riccati equations. International Journal of Control 2004; 77:767-788.

12. Kressner D. Numerical Methods for General and Structured Eigenvalue Problems. Lecture Notes in Computational Science and Engineering, vol. 46. Springer: Berlin, 2005.

13. Bittanti S, Colaneri P, De Nicolao G. The difference periodic Riccati equation for the periodic prediction problem. IEEE Transactions on Automatic Control 1988; 33:706-712.

14. Benner P, Byers R. Evaluating products of matrix pencils and collapsing matrix products. Numerical Linear Algebra with Applications 2001; 8:357-380.

15. Van Dooren P. Two point boundary value and periodic eigenvalue problems. Proceedings of the CACSD'99 Symposium, Kohala Coast, HI, 1999.

16. Oară C, Varga A. The general inner-outer factorization problem for discrete-time systems. Proceedings of the ECC'99, Karlsruhe, Germany, 1999.

17. Emami-Naeini A, Franklin GF. Deadbeat control and tracking of discrete-time systems. IEEE Transactions on Automatic Control 1982; 27:176-181. 


\section{PERIODIC RICCATI EQUATIONS}

18. Varga A, Van Dooren P. Computing the zeros of periodic descriptor systems. Systems and Control Letters 2003; 50:371-381.

19. Varga A. A periodic systems toolbox for MATLAB. Proceedings of IFAC 2005 World Congress, Prague, Czech Republic, 2005.

20. Bittanti S, Colaneri P, Guardabassi G. Analysis of the periodic Lyapunov and Riccati equations via canonical decomposition. SIAM Journal on Control and Optimization 1986; 24:1138-1149.

21. Hench JJ. Private communication, 1995.

22. Hairer E, Lubich C, Wanner G. Geometric Numerical Integration: Structure-Preserving Algorithms for Ordinary Differential Equations. Springer: Berlin, 2002.

23. Hairer E, Hairer M. GniCodes-MATLAB programs for geometric numerical integration. In Frontiers in Numerical Analysis Durham 2002, Blowey J et al. (eds). Springer: Berlin, 2003; 199-240.

24. Dieci L. Numerical integration of the differential Riccati equation and some related issues. SIAM Journal on Numerical Analysis 1992; 29:781-815.

25. Dieci L, Eirola T. Positive definiteness in the numerical solution of Riccati differential equations. Numerische Mathematik 1994; 67:303-313.

26. Varga A. Periodic Lyapunov equations: some applications and new algorithms. International Journal of Control 1997; 67:69-87.

27. Bittanti S, Colaneri P. Analysis of discrete-time linear periodic systems. In Digital Control and Signal Processing Systems and Techniques, Control and Dynamics Systems, vol. 78, Leondes CT (ed.). Academic Press: New York, 1996; 313-339.

28. Grasselli OM, Longhi S. Pole-placement for nonreachable periodic discrete-time systems. Mathematics of Control Signals and Systems 1991; 4:439-455.

29. Varga A. Computation of Kronecker-like forms of periodic matrix pairs. Proceedings of MTNS'04, Leuven, Belgium, 2004.

30. Varga A. Balancing related methods for minimal realization of periodic systems. Systems and Control Letters 1999; 36:339-349.

31. Van Dooren P. A generalized eigenvalue approach for solving Riccati equations. SIAM Journal on Scientific and Statistical Computing 1981; 2:121-135.

32. Golub GH, Van Loan CF. Matrix Computations. John Hopkins University Press: Baltimore, 1989.

33. Misra P, Van Dooren P, Varga A. Computation of structural invariants of generalized state-space systems. Automatica 1994; 30:1921-1936.

34. Byers R, Kressner D. Structured condition numbers for invariant subspaces. SIAM Journal on Matrix Analysis and Applications 2006; 28:326-347.

35. Varga A. Computation of Kalman decompositions of periodic systems. European Journal of Control 2004; 10:1-8.

36. Varga A, Pieters S. Gradient-based approach to solve optimal periodic output feedback control problems. Automatica 1998; 34:477-481.

37. Pittelkau ME. Optimal periodic control for spacecraft pointing and attitude determination. Journal of Guidance, Control, and Dynamics 1993; 16:1078-1084.

38. Colaneri P. Continuous-time periodic systems in $H_{2}$ and $H_{\infty}$. Part I: Theoretical aspects. Kybernetika 2000; 36:211-242.

39. Colaneri P. Continuous-time periodic systems in $H_{2}$ and $H_{\infty}$. Part II: State feedback problems. Kybernetika 2000; 36:329-350.

40. Sagfors MF, Toivonen HT, Lennartson B. State-space solution to the periodic multirate $H_{\infty}$ control problem: a lifting approach. IEEE Transactions on Automatic Control 2000; 45:2345-2350.

41. Van Dooren P. The computation of Kronecker's canonical form of a singular pencil. Linear Algebra and its Applications 1979; 27:103-141.

42. Oară C, Van Dooren P. An improved algorithm for the computation of structural invariants of a system pencil and related geometric aspects. Systems and Control Letters 1997; 30:39-48. 